\title{
Novel Relay Node Placement Algorithms for Establishing Connected Topologies
}

\author{
Fatih Senel ${ }^{1, *}$, Mohamed Younis ${ }^{2, *}$
}

\begin{abstract}
This paper addresses the problem of placing the least number of fixed-range relay nodes (RNs) in order to establish multi-hop paths between every pair of terminals. We derive an optimal solution for the case of three terminals and for the cases of more than 3 terminals, we present three novel heuristics, namely, Optimized Triangle Selection based on Minimum Spanning Tree Triangulation (OTS-MST), Incremental Optimization based on Delaunay Triangulation (IODT) and hybrid approach. These heuristics take advantage of the optimal threeterminal based solution by forming connected sub-graphs for steinerized sets of three terminals and then connecting these sub-graphs via steinerized edges. OTS-MST considers triangles that have two mst edges and picks the subset of these triangles which provides the highest reduction in the total number of required RNs as compared to a solution that is based on steinerized mst edges. IO-DT calculates the Delaunay triangulation of terminals and iterates over the formed triangles. In each iteration, the algorithm steinerizes a triangle as part of the final topology if selecting such a triangle reduces the RN count. Finally we consider a hybrid approach, which combines the strengths of OTS-MST and IO-DT. The performance of the proposed algorithms is validated through simulation.
\end{abstract}

\footnotetext{
${ }^{*}$ Corresponding author

Email addresses: fatih.senel@antalya.edu.tr (Fatih Senel), younis@cs.umbc.edu (Mohamed Younis)

${ }^{1}$ Dept. of Computer Engineering, Antalya International University, Dosemealti, Antalya, 07190, Turkey.

${ }^{2}$ Dept. of Electrical and Computer Engineering, University of Maryland Baltimore County, Baltimore, MD, 21250, USA.
}

Preprint submitted to Journal of $\mathrm{LAT}_{E} X$ Templates

March 17, 2016 
Keywords: Relay Node placement, Network connectivity, Topology management, Interconnecting wireless networks.

\section{Introduction}

There has been a massive growth in the use of networked devices in civil, scientific and military applications in recent years. In some of these applications need arises to interconnect a set of terminals to serve a certain mission while 5 avoiding major infrastructure investment. An example is when multiple data sources or standalone networks need to be federated in order to aggregate their services and efficiently handle emerging events such as search and rescue, disaster management, criminal hunting, etc. The federation is often enabled by placing relays that form paths between these sources/networks. Another example is when a wireless sensor network (WSN) gets partitioned due to a breakdown or damage of a subset of its nodes. WSNs often serve in inhospitable environments which make nodes susceptible to failure, e.g., due to detonation of explosives in a battle field, natural calamities, etc. 1]. The failed nodes may cause the WSN to be split into disjoint segments. Restoring connectivity among these WSN segments would be necessary for the network to become operational again. Deploying relay nodes (RNs) is the most popular approach 2, 3] for repairing the network topology. Given the limited accessibility, the least resources should be used/employed.

On the other hand, many applications serve in controlled setups in which deterministic positioning of nodes is pursued to meet the design requirements and provide the desired quality of service [4]. Examples of these applications include security surveillance, habitat monitoring, safety assessment of factory floor, hazard detection in urban area, etc. The controlled nature of the application allows pre-planning and careful selection of where to place nodes and how they may communicate. Usually area or landmark coverage is the main criterion for determining where sensor nodes should be. For establishing connectivity, relay nodes (RNs) are deployed to ensure that data can be shared. 
Determining the number and the position of RNs in the aforementioned application scenarios is usually subject to optimization. The application designer often likes to employ the least RNs to achieve the desired connectivity goal. In addition, the positioning of these RNs should establish, possibly, a multi-hop path between the terminals, e.g., data sources or network segments, subject to communication range constraints. Our problem can be thus formally defined as follows: "Given $n$ disjoint WSN segments what are minimum number and position of the relay nodes for establishing inter-segment connectivity?". This problem is equivalent to the Steiner Minimum Tree with Minimal Steiner Points and Bounded Edge-Length (SMT-MSPBEL), which is shown to be NP-Hard by Lin and Xue [5].

A number of heuristics have been proposed in the literature for solving the 40 SMT-MSPBEL problem. Some of these heuristics focus on reducing the number of RNs and form a minimum spanning tree (mst), where the node degree of the individual RNs is two [6, 7, 5]. Meanwhile, the focus of other work has been on the degree of connectivity of the formed WSN topology with less emphasis on the RN count [8, 9]. In this paper we present an algorithm which finds the least ${ }_{45} \mathrm{RN}$ count for forming SMT-MSPBEL for the special case of three terminals (i.e., a triangle) by either: (1) populating the RNs along mst edges, i.e. two side of the triangle, with a distance of $R$ units apart, where $R$ is the communication range of RNs, or (2) finding a point $x$ inside the triangle, which we call Discrete Fermat Point, and connecting the vertices of the triangle to $x$. Next we study the generalized problem for the case of $n$ terminals and present three novel algorithms, namely Optimized Triangle Selection based on mst triangulation (OTS-MST), Incremental Optimization based on Delaunay Triangulation (IO-DT) and a hybrid heuristic. These algorithms opt to minimize the required $\mathrm{RN}$ count and vary in the solution quality and runtime complexities.

The main idea behind the proposed algorithms is to optimally solve the SMTMSPBEL problem for the case of three terminals and try to find the minimal $\mathrm{RN}$ count for larger networks using subsets of three terminals. OTS-MST finds the mst of the terminals and identifies the set of triangles which contains two 
mst edges. The algorithm then finds adjacent triangles (i.e. the triangles sharing one mst edge) and forms a graph of triangles called Triangular Adjacency Graph (TAG) whose vertices represent triangles and edges denote adjacency relationships. Using TAG, the OTS-MST algorithm finds the best subset of non-adjacent triangles which provides the highest reduction in the total number of required relays as compared to an mst-based solution. The runtime complexity of OTS-MST is $O\left(n^{2}\right)$. Meanwhile, the IO-DT algorithm calculates the Delaunay triangulation (DT) of terminals and iterates over the formed triangles with the time complexity of $O\left(n^{2}\right)$. In each iteration, the algorithm steinerizes a triangle, i.e., form a Steiner tree of 3 vertices, as part of the final topology if selecting such a triangle provides a reduction in the total number of required relays as compared to an mst-based solution. Unlike OTS-MST, IO-DT does not restrict itself to triangles having two mst edges. The third algorithm is a hybrid heuristic that combines the strengths of both OTS-MST and IO-DT. The performance of all three heuristics is also validated through simulation and is shown to exceed contemporary heuristics in the literature.

The next section discusses related work. Section III describes the optimal solution for three terminals. Sections IV, V and VI describe the OTS-MST, IO-DT and hybrid approaches in detail, respectively. The runtime complexity and performance of the algorithms are analyzed in section VII. Section VIII presents the simulation results. Finally, section IX concludes the paper.

\section{Related Work}

The goal of the proposed algorithms is to place the minimum number of RNs in such a way that there exists a path between every pair of terminals and each hop in the path is less than or equal to the transmission range of the individual nodes. As we mentioned, this problem is shown to be NP-Hard by Lin and Xue [5]. They have further presented a polynomial time approximation algorithm that deploys RNs along the mst edges of terminals. The algorithm first constructs the complete graph of terminals and form mst using Kruskal's 
algorithm. RNs are populated along the tree edges at a distance of at most $R$ apart, where $R$ is the communication range of RNs. In [10], the authors proved that the algorithm yields a solution that is at most 4 times as much as the optimal value. In [6], the authors employ a three-step heuristic. In the first step, they connect the nodes where the distance between the nodes is less than or equal to $R$. In the second step, 3-stars are formed for each subset of three nodes $u, v, w$, for which there exists a point $s$ such that $s$ is at most $R$ units away from $u, v$, and $w$. Assuming that $\overline{u v}$ and $\overline{u w}$ are mst edges, a 3-star modifies the $m s t$ by replacing $\overline{u v}$ and $\overline{u w}$ with $\overline{s u}, \overline{s v}$ and $\overline{s w}$. In the last step the algorithm populates RNs along modified mst edges that are not substituted with 3-stars. In [6], the authors also presented a randomized algorithm that yields better results, yet it has $50 \%$ probability of convergence. A randomized algorithm does not necessarily guarantee the solution consistently. In this paper, we focus on the same problem studied in [5, 6, 10]. Unlike these publications, the proposed algorithms strive to find a subset of triangles and form triangular Steiner trees by connecting the vertices of each triangle at a point inside the triangle which will be called as Discrete Fermat Point. As we validate through simulation, the proposed algorithms significantly outperform those of [5, 6, 10]. In [11], we have studied the problem in the context of federating disjoint network segments and presented an algorithm called FeSTA with $O\left(n^{4}\right)$ runtime complexity. The algorithm iterates over all possible triangles and greedily selects triangles to apply Discrete Fermat Point optimization. The algorithms proposed in this paper, namely OTS-MST, IO-DT and Hybrid, are significantly less complex than FeSTA. A preliminary version of IO-DT algorithm was reported in [12]. This paper presents an additional scheme and extend IO-DT.

The focus of [8] and [9] is on a variant of the SMT-MSPBEL problem where additional metric is to be optimized. In [8], Lee and Younis strive to connect the 115 terminals inward so that the inter-terminal topology has a high node degree. They have proposed an algorithm called CORP that models the deployment area as equal-sized grid cells. CORP has two phases. In the first phase, the algorithm iteratively identifies border terminals and determines the best cell 
for deploying an $\mathrm{RN}$ based on the distance between the farthest terminals (or RN) from the centroid. The cells connecting multiple terminals are called junction cells. In the second phase, after all terminals are connected, the algorithm prunes redundant RNs. In [9] a bio-inspired heuristic called SpiderWeb has been proposed to form topologies that not only exhibit stronger connectivity but also achieve better sensor coverage and enable balanced distribution of traffic load on the employed relays. Unlike these algorithms, minimizing the number of RNs is the main objective in this paper.

In addition to establishing connected topologies, relay node placement has been studied for improving the performance in terms of other metrics [4]. Network lifetime has received the most attention among them. Depending on the transmission media, energy consumption for transmitting data is directly proportional to $d^{j}$ where $d$ is the distance and $j \geq 2[7]$. Due to this fact, long distance data transmission is very costly and stabling multi-RN paths is advantageous. In [13] the authors have studied this problem for both single-tiered and two-tiered WSN architectures. In single-tiered architectures, sensors transmit their own messages and also forward messages from other nodes. Meanwhile in two-tiered architectures nodes transmit only their own messages to a relay node or the base-station. Distinct polynomial-time approximation RN placement algorithms for one- and two-tiered architectures have been developed by modeling the network as a unit disc graph and identifying the best position of RNs based on overlapping unit-disc regions. In [7], the authors have studied the same problem assuming that relays and sensors have different transmission ranges. [14] opted to reduce the communication energy consumed by a gateway node in sending data to the base-station. The authors consider a twotiered network architecture where mobile sensors are assigned to a cluster that is led by an aggregation-and-forwarding node. The RN placement and energy provisioning problem is formulated as a mixed-integer nonlinear programming optimization. In [15], the authors studied a similar problem with the goal of reducing the system overhead in two-tiered network architectures. In order to minimize the energy consumption, the problem is first formulated as Relay Node 

and not just sensor-relay connectivity. 


\section{Solving SMT-MSPBEL of 3-Terminals}

As mentioned earlier the problem that we address in this paper is how to

180

connect $n$ disjoint WSN segments using the minimum number of relay nodes. Such a problem is equivalent to SMT-MSPBEL problem [5]. In this section we provide an optimal solution for forming SMT-MSPBEL for the special case of three terminals. In the following sections we will direct out attention to the generalized case for $n$ terminals. Before presenting the 3-terminals algorithm 185 we give some important definitions that cover the basis of the solution. Note that we assume that all relay and terminal nodes have the same communication range " $R$ ".

Definition 1. The minimum number of Steiner Points (SPs) required for forming SMT-MSPBEL for two terminals $u$ and $v$ is called the sp-weight of the edge $\overline{u v}$ and denoted as $W_{s p}(u, v)=\left\lceil\frac{|u v|}{R}\right\rceil-1$, where $|u v|$ is the Euclidean distance between $u$ and $v$.

Definition 2. Let $T_{i}$ be a triangle where the vertices are $(u, v, w)$. The Fermat Point (FP) $f_{i}$ (also called Torricelli Point [24]) for the triangle $T_{i}$ is the point that minimizes the sum of Euclidean distances from the vertices of $T_{i}$ to $f_{i}$, i.e., $f_{i}=\min _{q}\{|u q|+|v q|+|w q|\}$ where $q$ is any arbitrary point in triangle $T_{i}$.

Lemma 1. If a triangle $T_{i}$ contains an angle greater than or equal to $120^{\circ}$, then FP of $T_{i}$ is located at the corner with the biggest angle. [24]

Definition 3. Let $T_{i}$ be the triangle $(u, v, w)$ and $q$ be a point inside $T_{i}$. The 200 number of relays required for connecting the vertices of $T_{i}$ at an arbitrary point $q$ is called the fp-weight of $T_{i}$ at $q$ and denoted as $W_{f p}\left(T_{i}, q\right)$. Basically, a relay will be placed at $q$ and then connected to the vertices, $u, v$ and $w$, by placing the relays on the edges $\overline{q u}, \overline{q v}$, and $\overline{q w}$, respectively. Thus, $W_{f p}\left(T_{i}, q\right)=$ $W_{s p}(u, q)+W_{s p}(v, q)+W_{s p}(w, q)+1$, or

$$
W_{f p}\left(T_{i}, q\right)=\left(\left\lceil\frac{|u q|}{R}\right\rceil-1\right)+\left(\left\lceil\frac{|v q|}{R}\right\rceil-1\right)+\left(\left\lceil\frac{|w q|}{R}\right\rceil-1\right)+1
$$




$$
W_{f p}\left(T_{i}, q\right)=\left\lceil\frac{|u q|}{R}\right\rceil+\left\lceil\frac{|v q|}{R}\right\rceil+\left\lceil\frac{|w q|}{R}\right\rceil-2
$$

The point $\varphi_{i}$ that minimizes the fp-weight of triangle $T_{i}$ is called Discrete Fermat Point (DFP) and the weight at DFP is called the $d f p$-weight of the triangles and denoted as $W_{f p}\left(T_{i}, \varphi_{i}\right)$, or simply $W_{d f p}\left(T_{i}\right)$. DFP can be formally defined as follows:

$$
\varphi_{i}=\min _{q}\left\{\left\lceil\frac{|u q|}{R}\right\rceil+\left\lceil\frac{|v q|}{R}\right\rceil+\left\lceil\frac{|w q|}{R}\right\rceil-2\right\}
$$

If we remove the ceiling brackets and multiply by " $R$ ", the minimization becomes linear in the distance and the point $\varphi_{i}$ would be the Fermat Point. The DFP is the discretized version of Fermat Point, which is the basis for the name. There are only two possible ways to form optimal SMT-MSPBEL for the three terminals $u, v$, and $w$ : (1) via steinerizing the two smallest edges of the triangle $T_{i}(u, v, w)$, as illustrated in Figure 1(a). This is also equivalent to forming steinerized $m s t$ for three terminals; (2) via finding DFP $\varphi_{i}$ of $T_{i}$ and steinerizing the edges $\overline{u \varphi_{i}}, \overline{v \varphi_{i}}, \overline{w \varphi_{i}}$ as illustrated in Figure 1(b). As we are going to explain later, the solution of steinerizing the two smallest edges, i.e., Figure 1(a), corresponds to having DFP located on one of the sides on the triangle.

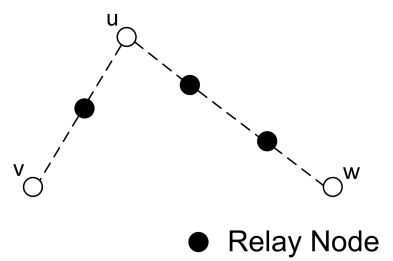

(a)

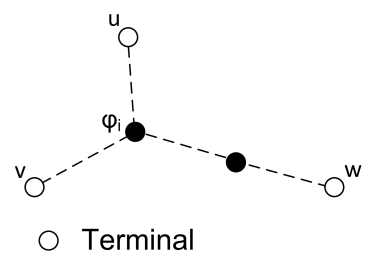

(b)

Figure 1: Two possible solutions for forming SMT-MSPBEL for a triangle $T_{i}=(u, v, w)$, (a) via steinerizing the two smallest edges of the triangle, (b) via DFP $\varphi_{i}$

Theorem 1. The optimal solution for forming SMT-MSPBEL for three terminals $u, v$, and $w$ can be achieved either via steinerizing the two smallest edges 


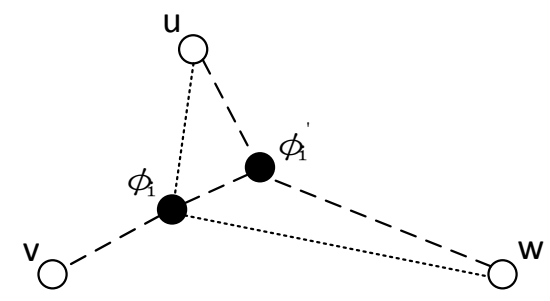

Figure 2: Illustrating how to form SMT-MSPBEL via DFP for a sub-triangle $T_{i}^{\prime}\left(u, \varphi_{i}, w\right)$

of the triangle $T_{i}(u, v, w)$ or steinerizing the edges from its vertices to the DFP $\varphi_{i}$

Proof. We prove the theorem in two cases:

Case 1: In the optimal solution all RNs lie on the straight line joining pairs of terminals. This case is straightforward and applicable if there is no such point $T_{i}$ inside the triangle which provides a better solution.

Case 2: In the optimal solution there is a RN inside the triangle which connects 225 all pairs of terminals. By definition 3 we know that such an $\mathrm{RN}$ is located at the DFP of the triangle. As a result of finding the DFP $\varphi_{i}$ of a triangle, three sub-triangles are formed namely, $T_{i}^{\prime}\left(u, \varphi_{i}, w\right), T_{i}^{\prime \prime}\left(u, \varphi_{i}, v\right)$ and $T_{i}^{\prime \prime \prime}\left(v, \varphi_{i}, w\right)$.

To form SMT-MSPBEL via DFP for $T_{i}$, we form steinerized $m s t$ for each of these sub-triangles. To prove the optimality, we need to show that forming the steinerized $m s t$ is the optimal solution for the sub-triangles. We pursue a proof by contradiction. Suppose forming SMT-MSPBEL for a sub-triangle, say $T_{i}^{\prime}\left(u, \varphi_{i}, w\right)$, via DFP yields a better solution than forming steinerized $m s t$ using $\varphi_{i}^{\prime}$, the DFP of the sub-triangle $T_{i}^{\prime}\left(u, \varphi_{i}, w\right)$, as illustrated in Figure 2 Based on that assumption we can write the following: $W_{f p}\left(T_{i}^{\prime}, \varphi_{i}^{\prime}\right)<W_{s p}\left(u, \varphi_{i}\right)+$ $W_{s p}\left(w, \varphi_{i}\right)$

$$
\left\lceil\frac{\left|\varphi_{i} \varphi_{i}^{\prime}\right|}{R}\right\rceil+\left\lceil\frac{\left|u \varphi_{i}^{\prime}\right|}{R}\right\rceil+\left\lceil\frac{\left|w \varphi_{i}^{\prime}\right|}{R}\right\rceil-2<\left\lceil\frac{\left|u \varphi_{i}\right|}{R}\right\rceil+\left\lceil\frac{\left|w \varphi_{i}\right|}{R}\right\rceil-2
$$

In addition applying triangular inequality on $T\left(v, \varphi_{i}, \varphi_{i}^{\prime}\right)$, we have: $\left|v \varphi_{i}\right|+$ 
$\left|\varphi_{i} \varphi_{i}^{\prime}\right| \geq\left|v \varphi_{i}^{\prime}\right|$. Then, $\frac{\left|v \varphi_{i}\right|}{R}+\frac{\left|\varphi_{i} \varphi_{i}^{\prime}\right|}{R} \geq \frac{\left|v \varphi_{i}^{\prime}\right|}{R}$ and thus,

$$
\left\lceil\frac{\left|v \varphi_{i}\right|}{R}\right\rceil+\left\lceil\frac{\left|\varphi_{i} \varphi_{i}^{\prime}\right|}{R}\right\rceil \geq\left\lceil\frac{\left|v \varphi_{i}^{\prime}\right|}{R}\right\rceil
$$

After forming SMT-MSPBEL for the sub-triangle $T_{i}^{\prime}\left(u, \varphi_{i}, w\right)$ via DFP, the total number of relay nodes required for forming SMT-MSPBEL for triangle $T_{i}(u, v, w)$ is the sum of sp-weights of the edges $\overline{v \varphi_{i}}, \overline{\varphi_{i} \varphi_{i}^{\prime}}, \overline{u \varphi_{i}^{\prime}}, \overline{w \varphi_{i}^{\prime}}$ plus 2 for the points $\varphi_{i}$ and $\varphi_{i}^{\prime}$

$$
\begin{aligned}
& \left(\left\lceil\frac{\left|v \varphi_{i}\right|}{R}\right\rceil-1\right)+\left(\left\lceil\frac{\left|\varphi_{i} \varphi_{i}^{\prime}\right|}{R}\right\rceil-1\right)+\left(\left\lceil\frac{\left|u \varphi_{i}^{\prime}\right|}{R}\right\rceil-1\right)+\left(\left\lceil\frac{\left|w \varphi_{i}^{\prime}\right|}{R}\right\rceil-1\right)+2 \\
& =\left\lceil\frac{\left|v \varphi_{i}\right|}{R}\right\rceil+\left\lceil\frac{\left|\varphi_{i} \varphi_{i}^{\prime}\right|}{R}\right\rceil+\left\lceil\frac{\left|u \varphi_{i}^{\prime}\right|}{R}\right\rceil+\left\lceil\frac{\left|w \varphi_{i}^{\prime}\right|}{R}\right\rceil-2
\end{aligned}
$$

Using (4) we have,

$$
\begin{gathered}
\left\lceil\frac{\left|v \varphi_{i}\right|}{R}\right\rceil+\left\lceil\frac{\left|\varphi_{i} \varphi_{i}^{\prime}\right|}{R}\right\rceil+\left\lceil\frac{\left|u \varphi_{i}^{\prime}\right|}{R}\right\rceil+\left\lceil\frac{\left|w \varphi_{i}^{\prime}\right|}{R}\right\rceil-2 \geq \\
\left\lceil\frac{\left|v \varphi_{i}^{\prime}\right|}{R}\right\rceil+\left\lceil\frac{\left|u \varphi_{i}^{\prime}\right|}{R}\right\rceil+\left\lceil\frac{\left|w \varphi_{i}^{\prime}\right|}{R}\right\rceil-2
\end{gathered}
$$

Based on definition 3. $\left\lceil\frac{\left|v \varphi_{i}^{\prime}\right|}{R}\right\rceil+\left\lceil\frac{\left|u \varphi_{i}^{\prime}\right|}{R}\right\rceil+\left\lceil\frac{\left|w \varphi_{i}^{\prime}\right|}{R}\right\rceil-2$ is the fp-weight of the triangle $T_{i}(u, v, w)$ at point $\varphi_{i}^{\prime}$, which can be shortly written as $W_{f p}\left(T_{i}, \varphi_{i}^{\prime}\right)$

$$
\left\lceil\frac{\left|v \varphi_{i}\right|}{R}\right\rceil+\left\lceil\frac{\left|\varphi_{i} \varphi_{i}^{\prime}\right|}{R}\right\rceil+\left\lceil\frac{\left|u \varphi_{i}^{\prime}\right|}{R}\right\rceil+\left\lceil\frac{\left|w \varphi_{i}^{\prime}\right|}{R}\right\rceil-2 \geq W_{f p}\left(T_{i}, \varphi_{i}^{\prime}\right)
$$

Using (3) we can write

$$
\begin{gathered}
W_{d f p}\left(T_{i}\right)=\left\lceil\frac{\left|u \varphi_{i}\right|}{R}\right\rceil+\left\lceil\frac{\left|v \varphi_{i}\right|}{R}\right\rceil+\left\lceil\frac{\left|w \varphi_{i}\right|}{R}\right\rceil-2> \\
\left\lceil\frac{\left|v \varphi_{i}\right|}{R}\right\rceil+\left\lceil\frac{\left|\varphi_{i} \varphi_{i}^{\prime}\right|}{R}\right\rceil+\left\lceil\frac{\left|u \varphi_{i}^{\prime}\right|}{R}\right\rceil+\left\lceil\frac{\left|w \varphi_{i}^{\prime}\right|}{R}\right\rceil-2
\end{gathered}
$$

From (5) and (6), we have $W_{d f p}\left(T_{i}\right)>W_{f p}\left(T_{i}, \varphi_{i}^{\prime}\right)$. However this is a contra235 diction since the definition of DFP implies that $W_{d f p}\left(T_{i}\right)$ is minimal and cannot be greater than $W_{f p}\left(T_{i}, \varphi_{i}^{\prime}\right)$. Therefore $\varphi_{i}^{\prime}$ of $T_{i}^{\prime}\left(u, \varphi_{i}, w\right)$ must be equal to $\varphi_{i}$, which is equivalent for forming steinerized $m s t$ for the sub-triangle $T_{i}^{\prime}\left(u, \varphi_{i}, w\right)$. The same proof can be applied to $T_{i}^{\prime \prime}\left(u, \varphi_{i}, v\right)$ and $T_{i}^{\prime \prime \prime}\left(v, \varphi_{i}, w\right)$. For complete- 
ness, it is worth noting that if we consider simultaneously steinerizing two of the sub-triangles via DFP, the resulting topology will not be cycle-free. Therefore, considering two (or three) triangles simultaneously will increase the required number of RNs and provide no benefit to the solution.

Per theorem 1, assuming that steinerizing the two smallest mst edges does not provide a better solution, if we find the DFP $\varphi_{i}$ of a triangle $T_{i}$, we find the optimal SMT-MSPBEL for $T_{i}$. The rest of this section describes how to find the DFP $\varphi_{i}$ of a triangle $T_{i}$.

Theorem 2. Let $T_{i}(u, v, w)$ be a triangle, $f_{i}$ be the Fermat Point of $T_{i}$. For any arbitrary point $q, W_{f p}\left(T_{i}, q\right) \geq W_{f p}\left(T_{i}, f_{i}\right)-2$

Proof. Let $D\left(T_{i}, q\right)=\frac{|u q|+|v q|+|w q|}{R}$, and $E\left(T_{i}, q\right)=\left\lceil\frac{|u q|}{R}\right\rceil+\left\lceil\frac{|v q|}{R}\right\rceil+\left\lceil\frac{|w q|}{R}\right\rceil$ where $R$ is constant. Per definition 3 we can write

$$
D\left(T_{i}, q\right) \leq E\left(T_{i}, q\right)
$$

Moreover we have,

$$
0 \leq E\left(T_{i}, q\right)-D\left(T_{i}, q\right)<3
$$

Per definition 2 we know, $D\left(T_{i}, q\right) \geq D\left(T_{i}, f_{i}\right)$ and the equality only holds when $q=f_{i}$ which is a unique point. So let's say $q \neq f_{i}$, then $D\left(T_{i}, q\right)>D\left(T_{i}, f_{i}\right)$. Now let's say $D\left(T_{i}, q\right)=E\left(T_{i}, q\right)-\epsilon_{0}$, and $D\left(T_{i}, f_{i}\right)=E\left(T_{i}, f_{i}\right)-\epsilon_{1}$, where $0 \leq \epsilon_{0}, \epsilon_{1}<3$. Since $D\left(T_{i}, q\right)>D\left(T_{i}, f_{i}\right)$, we can write

$$
E\left(T_{i}, q\right)-\epsilon_{0}>E\left(T_{i}, f_{i}\right)-\epsilon_{1}
$$

Thus

$$
E\left(T_{i}, q\right)>E\left(T_{i}, f_{i}\right)-\left(\epsilon_{1}-\epsilon_{0}\right)
$$

We know that $0 \leq \epsilon_{0}, \epsilon_{1}<3$, it is clear that $3>\epsilon_{1}-\epsilon_{0}$. Therefore we have 250 $E\left(T_{i}, q\right)>E\left(T_{i}, f_{i}\right)-3$. Since $E\left(T_{i}, q\right)=W_{f p}\left(T_{i}, q\right)+2$ and $W_{f p}$ is an integervalued function, we can write $W_{f p}\left(T_{i}, q\right) \geq W_{f p}\left(T_{i}, f_{i}\right)-2$ 
In theorem 2, we proved a lower bound for the fp-weight of any arbitrary point $q$. The theorem 2 implies that the $d f p$-weight of a triangle cannot be less than $W_{f p}\left(T_{i}, f_{i}\right)-2$. We can write the following: $|u q|=k_{1} R+\epsilon_{1},|v q|=k_{2} R+\epsilon_{2}$, ${ }_{255}$ and $|w q|=k_{3} R+\epsilon_{3}$ where $k_{i} \in \mathbb{Z}$ and $\epsilon_{i}<R, \forall i \in 1,2,3$. We thus can re-write (2) as:

$$
\varphi_{i}=\min _{q} k_{1}+k_{2}+k_{3}+\left\{\left\lceil\frac{\epsilon_{1}}{R}\right\rceil+\left\lceil\frac{\epsilon_{2}}{R}\right\rceil+\left\lceil\frac{\epsilon_{3}}{R}\right\rceil-2\right\}
$$

The optimal choice of $\varphi_{i}$ corresponds to $\epsilon_{i}=0, \forall i \in 1,2,3$, which will be equivalent to the Fermat Point of the triangle. If $\varphi_{i}$ does not match the Fermat point, we should have either $\epsilon_{1}=\epsilon_{2}=0$, or $\epsilon_{1}=\epsilon_{3}=0$, or $\epsilon_{2}=\epsilon_{3}=0$, in order to minimize (17). Given that $|u q|=k_{1} R+\epsilon_{1}$ and $|v q|=k_{2} R+\epsilon_{2}$, if a choice for the point $q$ that makes $\epsilon_{1}=\epsilon_{2}=0$ will be located at the intersection of two circles; one is centered at $u$ with radius $k_{1} R$, the other is centered at $v$ with radius $k_{2} R$. The same applies for $\epsilon_{1}=\epsilon_{3}=0$, and $\epsilon_{2}=\epsilon_{3}=0$.

At this point we are dealing with how to find DFP, in essence how many RNs can be saved by moving $f_{i}$ to another location. Due to theorem 2 our saving can be at most 2. Figure 3 (a), (b) and (c) analyzes the cases for how DFP is obtained starting from Fermat Point $f_{i}$ and moving $f_{i}$ to the circle intersections. In Figure 3 two circles are drawn from each corner in such a way that FP $f_{i}$ is located inside the bigger circle, but outside the smaller circle. For example in Figure 3(a), assuming that $\left|u f_{i}\right|=k_{1} R+\epsilon_{1}$ where $0 \leq \epsilon_{1}<R$, two circles are drawn which are both centered at $u$ where the bigger circle have a radius $\left(k_{1}+1\right) R$ and smaller circle have a radius $k_{1} R$. The other circles are drawn in the same manner from other corners $v$ and $w$. In Figure 3(a) the points in the shaded region have a $d f p$-weight that is 2 less than the weight at $f_{i}$ which is optimal according to theorem 2. If there is no region where $2 \mathrm{RNs}$ can be saved in that case we will look whether we can save 1 RN. Figure 3(b) illustrates the case where at most $1 \mathrm{RN}$ can be saved which is the intersection points of circles. Figure 3(c) illustrates the case when no RN can be saved. In Figure 3(c) since the angle $\widehat{a c b}$ is greater than $120^{\circ}, f_{i}$ of the triangle is located on the corner 


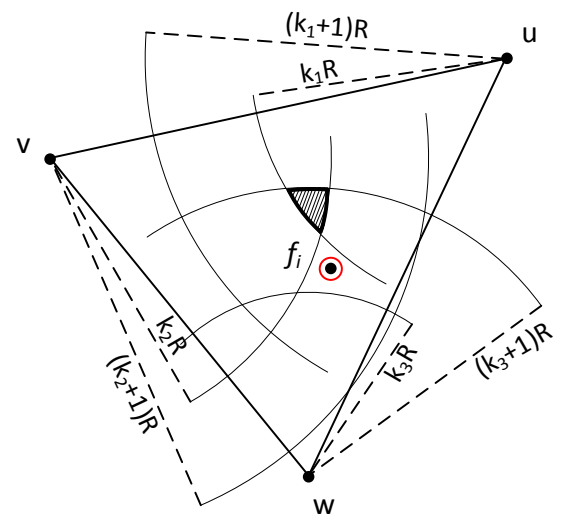

(a)

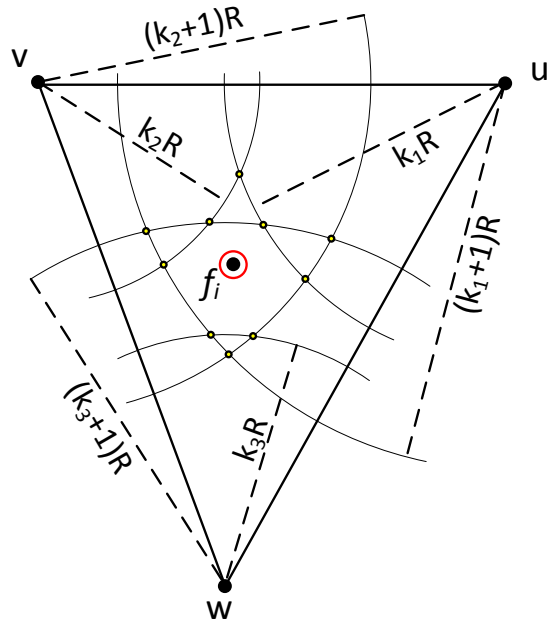

(b)

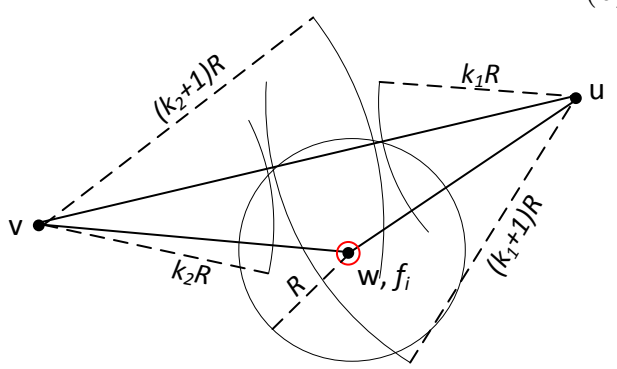

(c)

Figure 3: Case-by-case analysis of the affect of moving $f_{i}$ to circle intersections. (a) The $d f p$-weights of the points in the shaded region are 2 less than the weight of $f_{i}$. The shaded region is the intersection of circles centered at $u, v$ and $w$ having radii, $k_{1} R, k_{2} R$ and $\left(k_{3}+1\right) R$, respectively. (b) The $d f p$-weights of the intersections points are 1 less than the weight $f_{i}$, (c) No possible gain.

of the largest angle (i.e., $f_{i}=w$ ). In this case when we draw circles centered at the corners $u$ and $v$ none of the intersections provide a better solution than $f_{i}$. At this point one can ask the following question: How can we make sure that these 3 cases reflect all possibilities? In other words, in the case that we save $1 \mathrm{RN}$ as illustrated in Figure 3 (b) (or no RNs as in Figure 3 (c), is there another point somewhere else which is far from $f_{i}$ where we can save 2 RNs? As we discussed above the points that make $\epsilon_{1}=\epsilon_{2}=0$ are located at the intersection of two circles; one is centered at $u$ with radius $k_{1} R$, the other is centered at $v$ with radius $k_{2} R$. To find all these points which make $\epsilon_{1}=\epsilon_{2}=0$ 


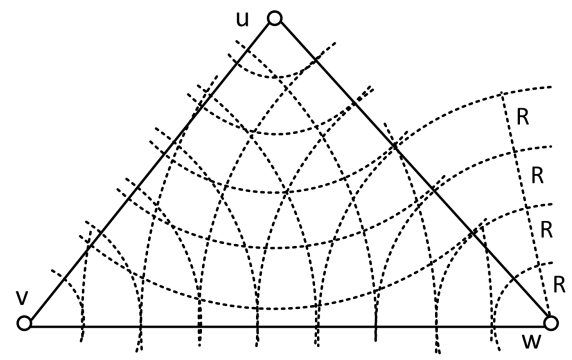

Figure 4: The intersection points of the circles which are centered at the corners with incremental radii $(R ; 2 R ; 3 R ; ; k R)$

we draw circles with incremental radii $(R ; 2 R ; 3 R ; ; k R)$ from each corner, as illustrated in Figure 4. As shown in the figure, the DFP can be located at one of the edges. The intersection points which are far from $f_{i}$ and close to one of the corners (lets say $u$ ), also satisfy $\epsilon_{1}=\epsilon_{2}=0$ property. In this case, however, $k_{1}$ decreases while $k_{2}$ and $k_{3}$ increases. In other words, we save from one of the corners at an increased cost from the other two corners. Thus the overall saving will converge to one of the cases which is illustrated in Figure 3. Therefore, we conclude that no further optimization is possible. In addition, since two circles are drawn for each corner of the triangle, the maximum number of intersection points of the circles will be constant. Hence the time complexity of finding DFP for a triangle will be $O(1)$. The pseudo-code for finding the DFP of triangle is provided in Algorithm 1 .

Theorem 1 provides the base for finding the optimal solution for the SMTMSPBEL for the case of 3 terminals. Since SMT-MSPBEL is fundamentally NP-hard for the general case of $n$ terminals, we propose three novel heuristics by grouping the $n$ terminals into a set of non-collinear 3 -tuples, forming a SMT-MSPBEL for each 3-tuple, and then establishing a connected topology by federating these formed 3-tuples based SMT-MSPBELs. The differences between the proposed heuristics are in how the set of 3-tuples are picked among all $C_{3}^{n}$ possible combinations and how the formed 3-tuples based SMT-MSPBELs are interconnected. The proposed heuristics are presented in the next sections. 


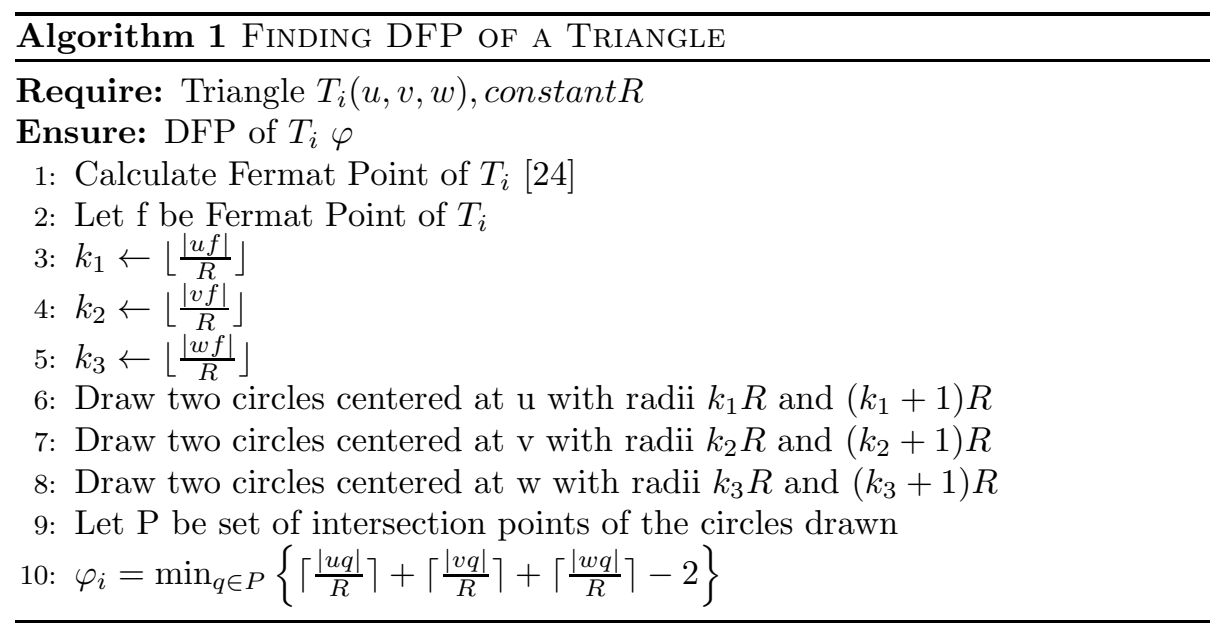

\section{Optimized Triangle Selection Based on mst Triangulation}

One of the approaches for establishing inter-terminal connectivity is to form steinerized $m s t$ where RNs are placed $R$ units apart from each other along the $m s t$ edges of the terminals [5]. However, in section 3 we have shown that steinerizing a set of 3 terminals, i.e., a triangle, by determining the DFP minimizes the required number of RNs. In this section we present an Optimized Triangle Selection based on mst triangulation (OTS-MST) algorithm that leverages the DFP based steinerization. Basically, the OTS-MST algorithm identifies a subset of triangles, i.e., all possible non-collinear 3 terminals, places RNs at the DFP of the picked triangles, modifies the mst of terminals on-the-fly and then forms steinerized mst on the modified tree where the required number of RNs is reduced. The details of the OTS-MST heuristic are provided in this section.

\subsection{OTS-MST Heuristic}

The main idea of the OTS-MST heuristic is to apply the DFP based steinerization, discussed in the previous section to a steinerized mst based solution of the SMT-MSPBEL problem. Basically, the triangles, i.e., sets of 3 terminals, that have two mst edges will be good candidates for consideration for 
the DFP-based optimization. In other words, these triangles become the set of 3-tuples for which optimal SMT-MSPBELs are formed, and the mst of the terminals will serve as the interconnection structure for these individual 3-tuple based SMT-MSPBELs. The fundamental question is whether some of these $m s t$-based triangles are redundant and if so, how to identify the best choices so that the least number of relays are involved in solving the SMT-MSPBEL problem for the entire set of terminals. The following definitions assist in answering these questions.

Definition 4. Let $T_{i}(u, v, w)$ be a triangle with two edges $e_{1}=\overline{u v}$ and $e_{2}=\overline{u w}$, that are on the mst of the set of terminals $V$. The mst-weight of $T_{i}$ is defined as $W_{m s t}\left(T_{i}\right)=W_{s p}\left(e_{1}\right)+W_{s p}\left(e_{2}\right)$. Later we will extend this definition for the case, when $T_{i}$ does not have edges on the mst of $V$.

Definition 5. The gain of $T_{i}$ is defined as the difference between mst- and dfpweights of $T_{i}$

$$
\operatorname{Gain}\left(T_{i}\right)=W_{m s t}\left(T_{i}\right)-W_{d f p}\left(T_{i}\right)
$$

Definition 6. The triangulation of a set of terminals $V$ based on mst edges in the $2 D$ plane is called mst triangulation and denoted as $\Gamma_{m s t}$. Let $G_{m s t}\left(V, E_{m s t}\right)$ be the minimum spanning tree of the terminals. A triangle $T_{i}(u, v, w) \in \Gamma_{m s t}$ if it satisfies the following properties: Let $\alpha$ be the $\widehat{u v w}$ angle where $0^{\circ}<\alpha<180^{\circ}$ and let $z$ be a point where $z \in V-\{u, v, w\}$ and $\overline{v z} \in E_{m s t}$. Assume that $\alpha^{\prime}$ and $\alpha^{\prime \prime}$ are the angles $\widehat{z v u}$ and $\widehat{z v w}$, respectively.

1. $\overline{u v} \in E_{m s t}$ and $\overline{v w} \in E_{m s t}$

2. $\alpha \neq \alpha^{\prime}+\alpha^{\prime \prime}$

Figure 5 illustrates the $m s t$ triangulation of the set of terminals $\{u, v, w, x, y, z\}$. Each triangle has two mst edges. The triangles $T(u, v, w)$ and $T(y, v, z)$ are not included in $\Gamma_{m s t}$ since their big angles are split by two other mst edges $\overline{v z}$ and $\overline{v u}$, respectively, which violates the second condition in definition 6 . The reason of excluding these triangles is to avoid redundant RN deployment. For 
example, the triangle $T(u, v, w)$ is split by an mst edge, namely $\overline{v z}$, into the triangles $T(u, v, z)$ and $T(v, w, z)$ that already cover the edges $\overline{v u}$ and $\overline{v w}$.

Redundancy in covering an mst

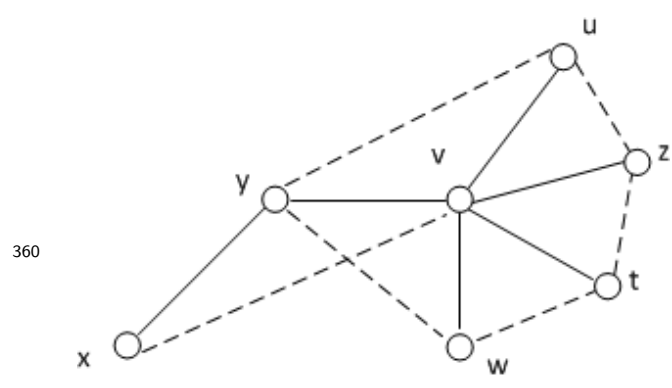

Figure 5: Illustrating mst triangulation, solid lines represent $m s t$ edges, dashed lines represent the additional edges introduced by mst tion in the relay count). As explained below. corresponding triangles share an mst edge. edge can take place when considering that edge in two triangles. For example, steinerizing the triangles $T(u, v, z)$ and $T(z, v, w)$ covers the mst edge $\overline{v z}$ twice. A more efficient way is to handle the edge $\overline{u v}$ in the triangle $T(u, v, y)$ and steinerize the triangle $T(z, v, w)$ to cover $\overline{v z}$. OTS-MST strives to avoid such redundancy by forming Triangular Adjacency Graph (TAG) and then using the TAG to find the subset of triangles which maximizes the total gain (reduc-

\subsubsection{Forming Triangular Adjacency Graph (TAG)}

As mentioned above, two triangles which share an mst edge spans 4 terminals. Therefore steinerizing both triangles will introduce redundancy since some nodes will be covered twice. In other words picking one triangle $T$ disqualifies all triangles that share an edge with $T$ and warrants their exclusion. The interesting question is how to select a set of triangles that ought to be steinerized in order to minimize the required relay count for federating all terminals. To be able to determine such a set, we introduce the Triangular Adjacency Graph (TAG). In TAG the vertices represent the triangles and the edges represent triangular adjacency, i.e., there is an edge between two vertices in TAG if the

Definition 7. Two triangles are called adjacent if they share an mst edge. Formally, let $T_{i}\left(u_{i}, v_{i}, w_{i}\right)$ and $T_{j}\left(u_{j}, v_{j}, w_{j}\right)$ be two triangles in $\Gamma_{m s t} . T_{i}$ and $T_{j}$ 
are called adjacent if the cardinality of the intersection of the vertices of the triangles is 2. $\left|\left\{u_{i}, v_{i}, w_{i}\right\} \cap\left\{u_{j}, v_{j}, w_{j}\right\}\right|=2$

The process of forming TAG is as follows. First, we perform mst triangulation of the terminals, i.e., form $\Gamma_{m s t}$, and then determine the gain for each triangle, as specified in definition 5 above. Each triangle in $\Gamma_{m s t}$ corresponds to a vertex in TAG and the weight of each vertex is the gain of the triangle, i.e., $c\left(T_{i}\right)=\operatorname{Gain}\left(T_{i}\right)$. An edge between two vertices in TAG is added if the corresponding triangles in $\Gamma_{m s t}$ are adjacent. In other words, let $G_{T}\left(V_{T}, E_{T}\right)$ be the TAG of $\Gamma_{m s t}$ where $V_{T}=\forall T_{i}$ s.t. $T_{i} \in \Gamma_{m s t}$ and $E=\forall\left(T_{i}, T_{j}\right)$ s.t. $T_{i}$ and $T_{j}$ are adjacent. The process of forming TAG is illustrated through an example in Figure 6.

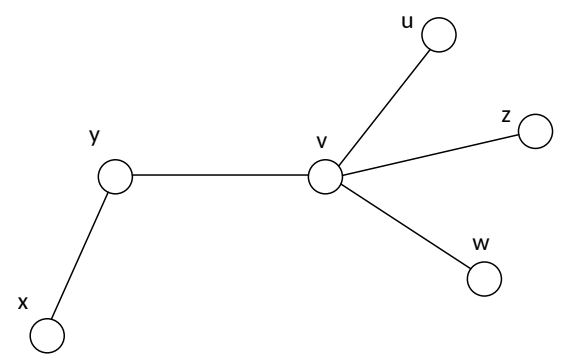

(a)

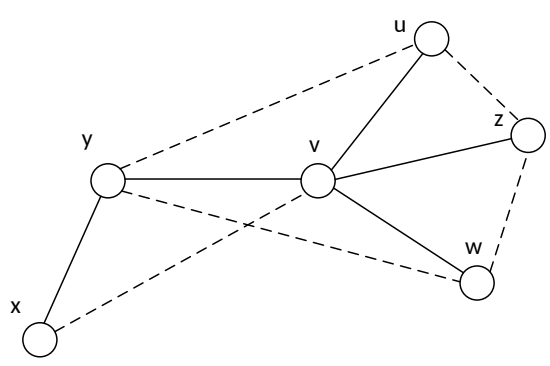

(b)

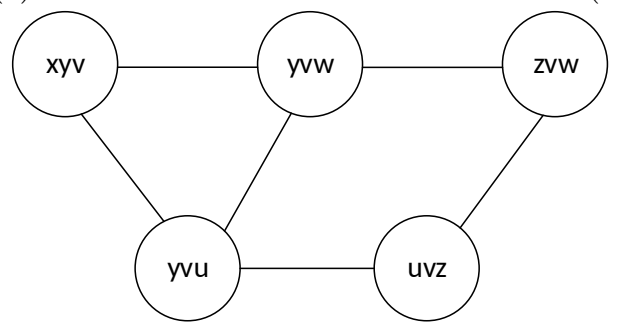

(c)

Figure 6: Forming TAG from mst. (a) mst of terminals. (b) $\Gamma_{m s t}$ of terminals. (c) corresponding TAG

As pointed out, the TAG will be used for selecting a set of non-adjacent triangles for optimization, i.e., forming SMT-MSPBEL via DFP. The terminals that are not covered by these selected non-adjacent triangles will be intercon- 
nected by steinerized mst edges. Obviously, the triangles of the highest gains will be preferred in order to reduce the overall RN count. Determining the set of non-adjacent triangles while maximizing the total gain is equivalent to finding maximum-weighted independent set $(M W I S)$ of vertices from TAG.

\subsubsection{Finding Max Weighted Independent Set (MWIS)}

The second step of the OTS-MST algorithm is to find the MWIS from TAG, which is also an NP-Hard problem. However, optimal solution can be found with dynamic programming (DP) if the graph is a tree [25]. The TAG, however, sets for the sub-trees rooted at $T_{k 1}, T_{k 2}, \cdots, T_{k m}$. On the other hand if $T_{k}$ is

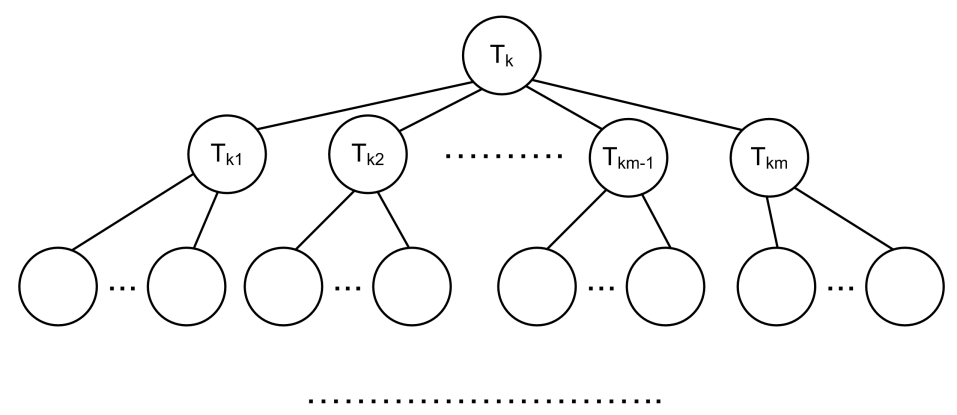

Figure 7: Illustrating triangular adjacency tree rooted at $T_{k}$. 
included in the independent set, none of the children of $T_{k}$ can be in optimal solution. Similarly the solution will be the union of $T_{k}$ and the independent sets for the sub-trees rooted at the grandchildren of $T_{k}$. We can formulate the problem as follows: Let $\mathcal{M}\left(T_{k}\right)$ be the optimal solution of $M W I S$ problem for the sub-tree rooted at $T_{k}$. We can write the following recursive formula.

$$
\mathcal{M}\left(T_{k}\right)=\max \left\{\left(\sum_{T_{i} \text { is child of } T_{k}} \mathcal{M}\left(T_{i}\right)\right), c\left(T_{k}\right)+\left(\sum_{T_{i} \text { is grandchild of } T_{k}} \mathcal{M}\left(T_{i}\right)\right)\right\}
$$

Due to ignoring some triangular adjacencies while breaking cycles, the resulting

${ }_{425}$ MWIS may not be actually independent and may still contain some adjacent triangles. Finally we identify the least weighted triangles (i.e., those providing the least gain as defined in Definition (5) which violate the independency condition in the resulting $M W I S$, and remove them from the MWIS.

After finding an MWIS as described above, the OTS-MST algorithm deploys RNs to the DFPs of the triangles in the MWIS and updates the mst of terminals on-the-fly. Let $T_{i}(u, v, w) \in M W I S$ where $\overline{u v}$ and $\overline{u w}$ are mst edges, and let $\varphi_{i}$ be the DFP of $T_{i}$. The algorithm updates mst by removing the two edges $\overline{u v}$ and $\overline{u w}$ and adding the three edges $\overline{u \varphi_{i}}, \overline{v \varphi_{i}}$ and $\overline{w \varphi_{i}}$ instead.

\subsection{Illustrative Example}

The pseudo-code for OTS-MST algorithm is provided in Algorithm 2 in the appendix. We illustrate how OTS-MST works with a detailed example. Let's assume we have 15 terminals, namely $\left\{t_{1}, t_{2}, \cdots, t_{15}\right\}$, whose $m s t$ is illustrated in Figure 8(a). The algorithm forms the triangular adjacency graph (TAG) of $\Gamma_{m s t}$ as shown in Figure $8(\mathrm{~b})$. Note that the triangle $\left\{t_{14}, t_{6}, t_{15}\right\}$ is 440 not in $\Gamma_{m s t}$, since the mst edge $\overline{t_{6} t_{2}}$ splits the obtuse angle $\widehat{t_{14} t_{6} t_{15}}$ into two. Some of the triangles in $\Gamma_{m s t}$ have zero-gain (dashed nodes in Figure $8(\mathrm{~b})$ ). Therefore the OTS-MST algorithm does not take these triangles into account, and consequently the dashed nodes and all induced edges can be pruned. The 


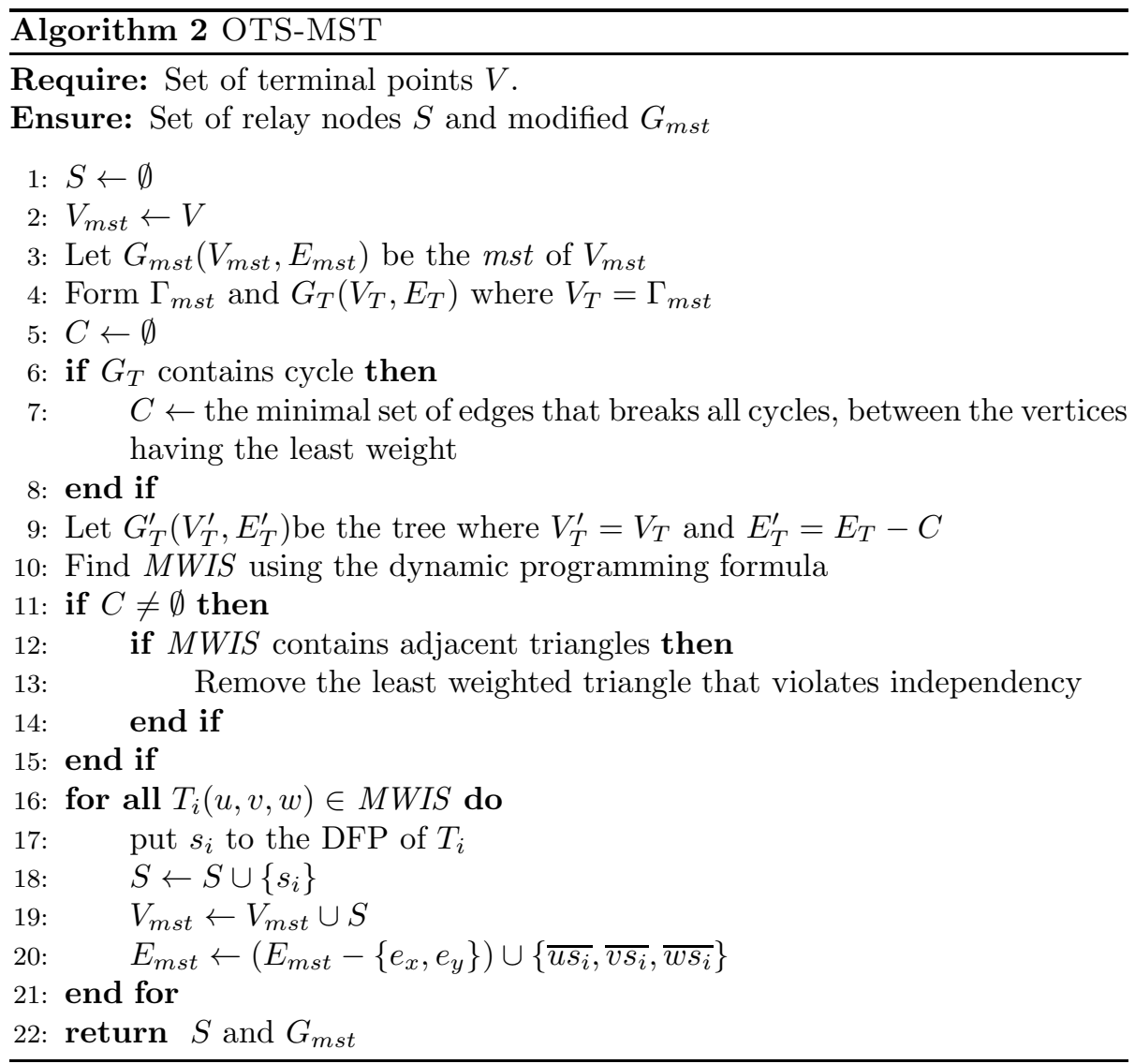

bold nodes in Figure 8(b) reflect the positive-gain triangles. In this example we assume the gain of all triangles which are shown in bold in Figure 8(b) is equal to 1. Pruning zero-gain triangles helps significantly for breaking the cycles in TAG. However, the remaining graph may still contain cycles. To apply dynamic programming we need to eliminate all cycles. When a cycle is detected, the algorithm breaks the cycles by ignoring some adjacencies as described above. In this example all edges are equivalent, i.e., each edge breaks only one cycle and the weights of adjacent vertices are the same. For this case, the algorithm randomly removes one of these edges to break the cycle. Let's say the algorithm ignored the edge $\overline{\left\{t_{14}, t_{6}, t_{2}\right\}\left\{t_{4}, t_{14}, t_{6}\right\}}$. After applying dynamic programming, we get $\left\{\left\{t_{2}, t_{8}, t_{12}\right\},\left\{t_{4}, t_{13}, t_{5}\right\},\left\{t_{4}, t_{14}, t_{6}\right\},\left\{t_{14}, t_{6}, t_{2}\right\}\right.$, 

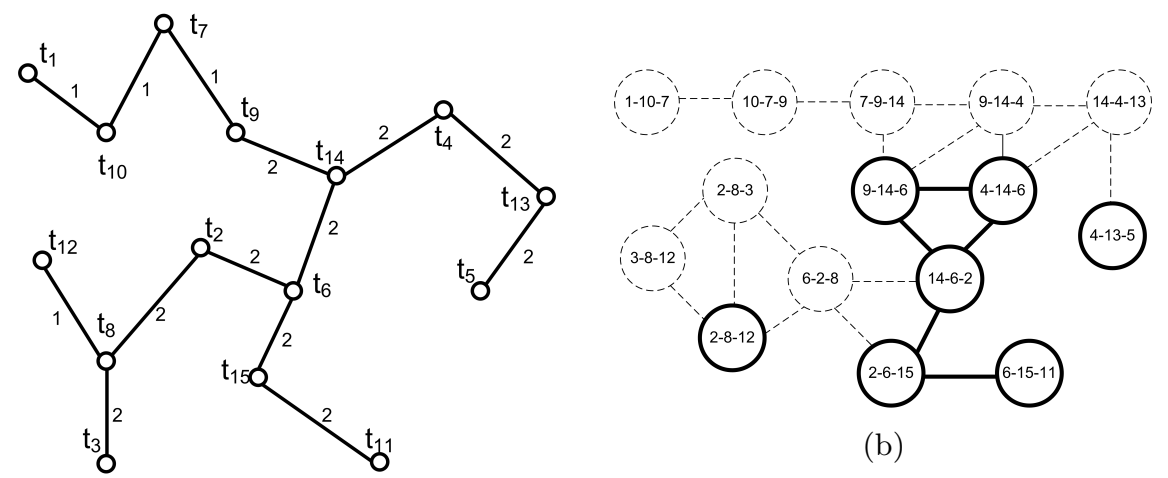

(b)

(a)

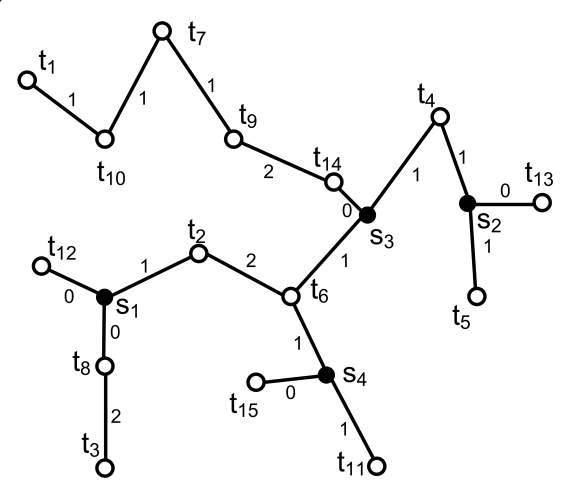

(c)

Figure 8: Illustration of how OTS-MST algorithm works. (a) mst of terminals, where the $s p$-weight of each edge is displayed in the figure. (b) The corresponding triangular adjacency graph (TAG). Solid vertices represent the triangles having positive gain. (c) Updated mst. The resulting SMT-MSPBEL will be formed steinerizing the mst edge where the number of required $\mathrm{RNs}$ is annotated on the edges.

$\left.\left\{t_{6}, t_{15}, t_{11}\right\}\right\}$ as the MWIS. Since $\left\{t_{4}, t_{14}, t_{6}\right\}$ and $\left\{t_{14}, t_{6}, t_{2}\right\}$ are adjacent, the algorithm randomly removes one of them from MWIS. Assuming the latter is removed, $M W I S=\left\{\left\{t_{2}, t_{8}, t_{12}\right\},\left\{t_{4}, t_{13}, t_{5}\right\},\left\{t_{4}, t_{14}, t_{6}\right\},\left\{t_{6}, t_{15}, t_{11}\right\}\right\}$. OTS-MST deploys 4 RNs $\left\{s_{1}, s_{2}, s_{3}, s_{4}\right\}$ to the DFP's of the triangles in MWIS as illustrated in Figure 8(c). Finally the mst edges are steinerized in order to form SMT-MSPBEL. 


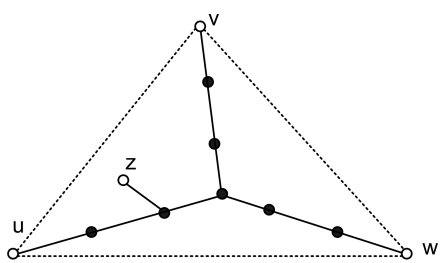

(a)

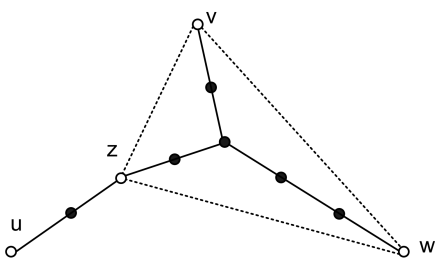

(b)

Figure 9: Illustrating redundant node deployment as a result of DFP optimization of a triangle having another terminal inside. (a) $7 \mathrm{RNs}$ are required (b) $6 \mathrm{RNs}$ are required for connecting 4 terminals.

\section{Incremental Optimization Based on Delaunay Triangulation (IO- DT)}

In OTS-MST, DFP optimization works for a small subset of triangles where the triangles have two mst edges. The main motivation of the IO-DT algorithm comes from the question on whether there are any other triangles not having two mst edges that could be optimized to further reduce the total number of RNs. Since there are $\left(\begin{array}{l}n \\ 3\end{array}\right)=O\left(n^{3}\right)$ possible triangles, where $n$ is the number of terminals, the complexity of the solution increases significantly if all triangles are to be considered. Most of these triangles, however, are not needed since considering big triangles having multiple terminals inside may require many redundant RNs. For example in Figure 9(a), the triangle $T(u, v, w)$ contains the terminal $z$. The DFP optimization of the triangle $T(u, v, w)$ yields $7 \mathrm{RNs}$ one of which is redundant as shown in Figure 9(b). Therefore the algorithm should avoid those redundancies. In other words we need a triangulation such that no terminal is located inside any triangle. Delaunay Triangulation (DT) meets this requirement and thus enables us to determine the set of triangles that ought to be considered. Hereafter, we use $\Gamma_{d t}$ to denote the triangles in the DT graph of terminals.

\subsection{The IO-DT Heuristic}

The IO-DT algorithm looks for the best option for connecting the terminals of each triangle in DT such that the required RN count for the entire network is minimized. It, however, tries to do so by incrementally considering groups of 
3 terminals. The first step for IO-DT is to determine $\Gamma_{d t}$ and then form $m s t$ of all terminals. IO-DT then considers each triangle in $\Gamma_{d t}$ to decide on whether mst edges or DFP based steinerization is better. In definition 4, we defined mst-weight of a triangle that contains two mst edges. In Delaunay Triangulation, however, a triangle may not contain two mst edges and thus we cannot apply definition 4 to calculate the mst-weight of the triangle. Definition 8 , is a generalization of definition 4 to handle these cases.

Definition 8. Let $T_{i}$ be a triangle with the vertices $(u, v, w)$. Given a minimum spanning tree $G_{m s t}$, assume that $\Upsilon_{u v}$ and $\Upsilon_{u w}$ are two paths in $G_{m s t}$ from u to $v$ and from $u$ to $w$, respectively, such that $\Upsilon_{u v} \nsubseteq \Upsilon_{u w}$ and $\Upsilon_{u v} \nsupseteq \Upsilon_{u w}$. Let $e_{x}$ and $e_{y}$ be the edges having the maximum sp-weight in $\Upsilon_{u v}$ and $\Upsilon_{u w}$, respectively. Also let $e_{z}$ be the mst edge having the maximum sp-weight in $\left(\Upsilon_{u v} \cup \Upsilon_{u w}\right)-$ $\left(\Upsilon_{u v} \cap \Upsilon_{u w}\right)$. The mst-weight of $T_{i}$ is denoted and computed as follows:

$$
W_{m s t}\left(T_{i}\right)= \begin{cases}W_{s p}\left(e_{x}\right)+W_{s p}\left(e_{y}\right), & \text { if } e_{x} \neq e_{y} \\ W_{s p}\left(e_{x}\right)+W_{s p}\left(e_{z}\right), & \text { otherwise }\end{cases}
$$

The two mst edges $e_{x}$ and $e_{y}$ (or $e_{z}$, if $e_{x}=e_{y}$ ) are called the associated mst edges with $T_{i}$. Finding mst-weight of $T_{i}$ is illustrated in Figure 10.

By definition 8 , in order to calculate the mst-weight of a triangle, we need to find two mst paths $\Upsilon_{u v}$ and $\Upsilon_{u w}$ and associated mst edges. Since the mst changes dynamically, i.e., after each RN deployment at a DFP of one of the triangles, the mst-weight and the gain of a triangle may change depending on the previously deployed RNs. To tackle these challenges, we pursue a greedy heuristic. Let $\Gamma_{d t}$ be the sorted list of triangles in the DT in an ascending order according to the $d f p$-weight of the individual triangles. The rationale for pursing such an order is that we'd like to introduce edges with the smallest possible $s p$ weight to the emerging network topology, i.e., involving the least $\mathrm{RN}$ count, so that we can remove existing edges with large sp-weight in subsequent iterations. 


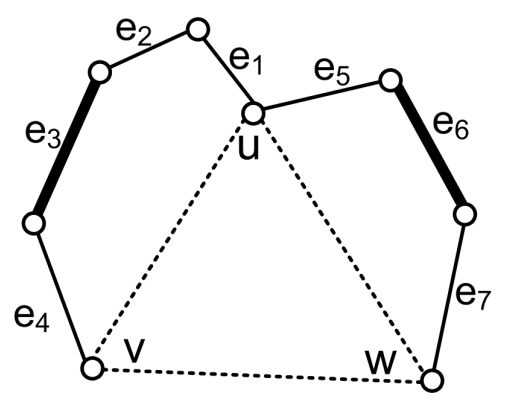

(a)

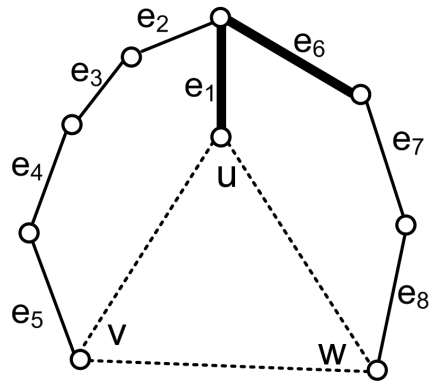

(b)

Figure 10: Illustration of calculating mst-weight of a triangle $T(u, v, w)$. Bold edges represent maximum sp-weighted mst edges in the paths $\Upsilon_{u v}$ and $\Upsilon_{u w}$. (a) $\Upsilon_{u v}=\left\{e_{1}, e_{2}, e_{3}, e_{4}\right\}$ and $\Upsilon_{u w}=\left\{e_{5}, e_{6}, e_{7}\right\}$ are node disjoint and $e_{3}$ and $e_{6}$ are maximum sp-weighted mst edges in $\Upsilon_{u v}$ and $\Upsilon_{u w}$, respectively (i.e. $e_{x}=e_{3}$ and $e_{y}=e_{6}$ ). Therefore, $W_{m s t}(T)=W_{s p}\left(e_{3}\right)+W_{s p}\left(e_{6}\right)$. (b) $\Upsilon_{u v}=\left\{e_{1}, e_{2}, e_{3}, e_{4}, e_{5}\right\}$ and $\Upsilon_{u w}=\left\{e_{1}, e_{6}, e_{7}, e_{8}\right\}$. Let $e_{1}$ is the maximum sp-weighted edge in both $\Upsilon_{u v}$ and $\Upsilon_{u w}$ (i.e. $e_{x}=e_{y}=e_{1}$ ), then we calculate maximum sp-weighted mst edge in $\left(\Upsilon_{u v} \cup \Upsilon_{u w}\right)-\left(\Upsilon_{u v} \cap \Upsilon_{u w}\right)=\left\{e_{2}, e_{3}, e_{4}, e_{5}, e_{6}, e_{7}, e_{8}\right\}$ which is $e_{6}$ in this example (i.e. $\left.e_{z}=e_{6}\right)$. Therefore $W_{m s t}(T)=W_{s p}\left(e_{1}\right)+W_{s p}\left(e_{6}\right)$

In other words, we would like to get rid of the edges having large sp-weight and keep the edges having small sp-weight in our final topology. The algorithm iteratively processes the triangles in $\Gamma_{d t}$. In each iteration the mst-weight and the gain of the selected triangle $T_{i}(u, v, w)$ are calculated. If $T_{i}$ has positive gain, the algorithm deploys an RN at the DFP of $T_{i}$, and updates the $m s t$ by removing the two mst edges that are associated with $T_{i}$ and adding the three edges $\overline{u \varphi_{i}}, \overline{v \varphi_{i}}$ and $\overline{w \varphi_{i}}$ instead, where $\varphi_{i}$ is the DFP of $T_{i}$.

\subsection{Illustrative Example}

The pseudo code for IO-DT algorithm is provided in Algorithm 3 in the appendix. Figure 11 illustrates how the IO-DT algorithm works through a detailed example. The configuration of the example is similar to that of Figure 8 First the algorithm calculates DT of the terminals. A subset of three terminals forms a triangle. The algorithm sorts the list of triangles in $\Gamma_{d t}$ according to their $d f p$-weights. Table 2 shows the sorted list of triangles. Figure 11(a) shows the DT of terminals. In Figure 11(a) the solid lines represent the mst edges and dashed lines are edges that belong only to $\Gamma_{d t}$. IO-DT is a greedy algorithm and at each iteration the algorithm picks the triangle having the 


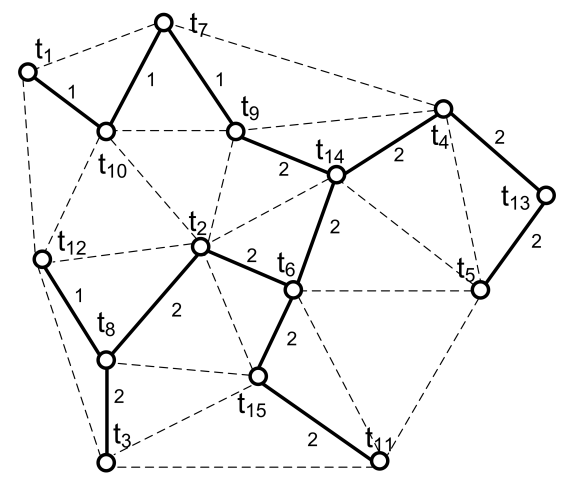

(a)

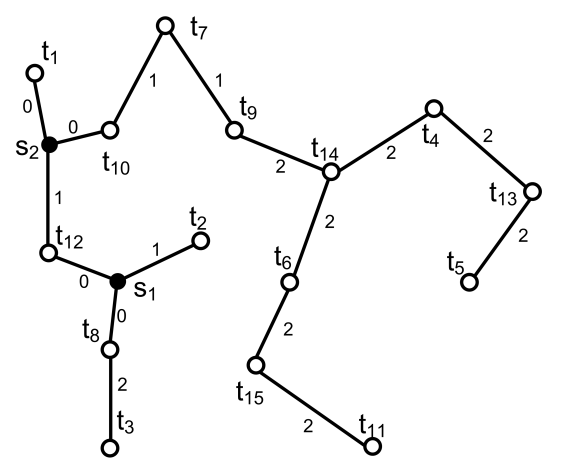

(c)

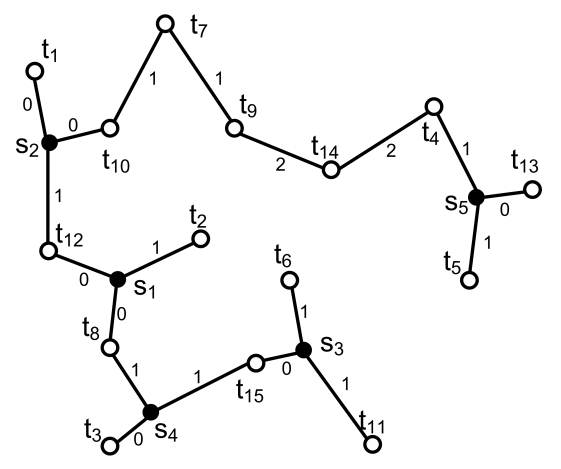

(e)

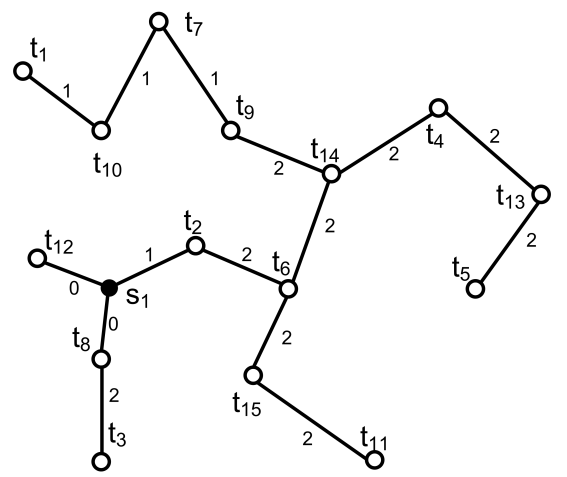

(b)

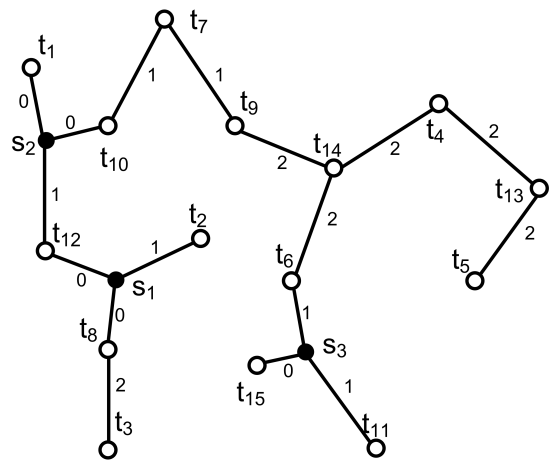

(d)

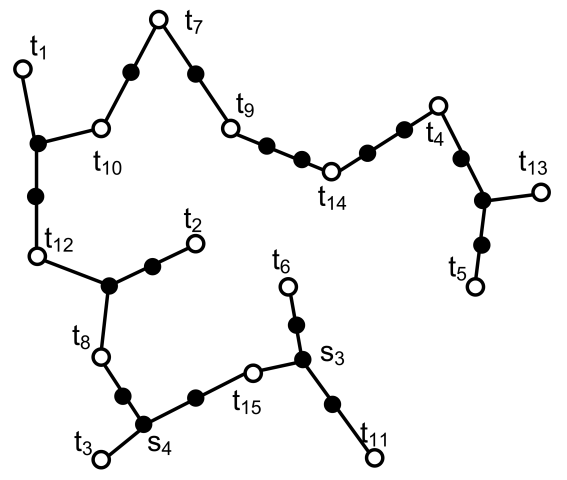

(f)

Figure 11: A Step-by-step illustration of how IO-DT algorithm works 


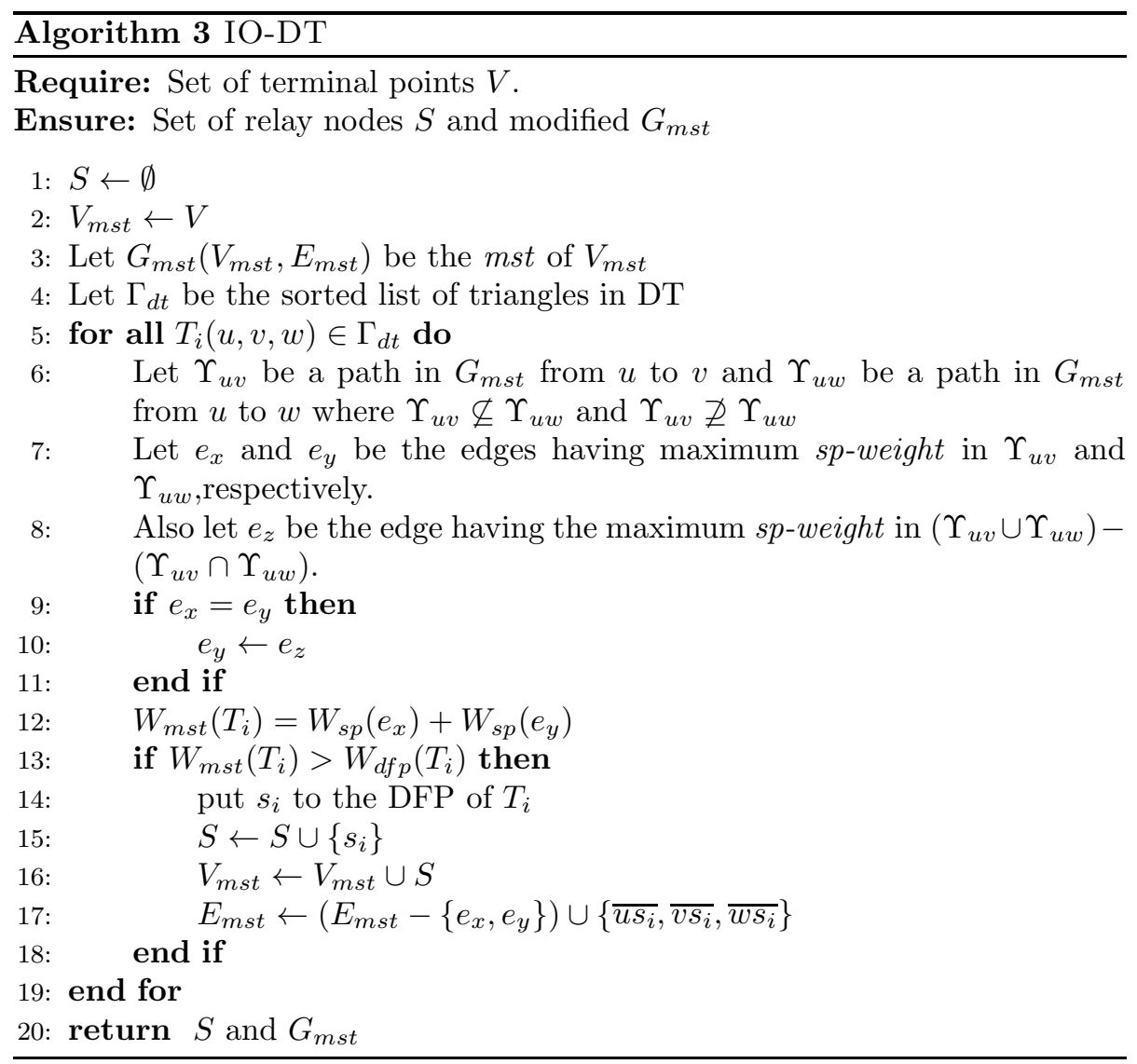

least $d f p$-weight. In the first iteration, the algorithm picks $T\left(t_{7}, t_{9}, t_{10}\right)$ and calculates the two paths $\Upsilon_{u v}$ and $\Upsilon_{u w}$ where $u=t_{7}, v=t_{9}$, and $w=t_{10}$. For this triangle, $\Upsilon_{u v}=\overline{t_{7} t_{9}}$ and $\Upsilon_{u} w=\overline{t_{7} t_{10}}$. Thus, $W_{m s t}\left(T\left(t_{7}, t_{9}, t_{10}\right)\right)=$ $W_{s p}\left(\overline{t_{7} t_{9}}\right)+W_{s p}\left(\overline{t_{7} t_{10}}\right)=2$. Since the $d f p$ - and mst-weights of the triangle $T\left(t_{7}, t_{9}, t_{10}\right)$ are equal, the algorithm skips this triangle. Similarly in the second iteration the triangle $T\left(t_{1}, t_{7}, t_{10}\right)$ is skipped due to the same reason. In the third iteration the triangle $T\left(t_{2}, t_{8}, t_{12}\right)$ is picked, where $\Upsilon_{u v}=\left\{\overline{t_{8} t_{2}}\right\}$ and $\Upsilon_{u w}=$ $\left\{\overline{t_{8} t_{12}}\right\}$ such that $u=t_{8}, v=t_{2}$, and $w=t_{12}$. Since the dfp-weight of the triangle is less than the mst-weight, the algorithm places an $\mathrm{RN}, s_{1}$, at the DFP of $T\left(t_{2}, t_{8}, t_{12}\right)$ and updates the $m s t$ as illustrated in Figure 11(b). In the next iteration the triangle $T\left(t_{1}, t_{10}, t_{12}\right)$ is considered, where $\Upsilon_{u v}=\left\{\overline{t_{1} t_{10}}\right\}$ and 

largest sp-weight in $\Upsilon_{u w}$, where $W_{m s t}\left(T\left(t_{1}, t_{10}, t_{12}\right)\right)=W_{s p}\left(\overline{t_{1} t_{10}}\right)+W_{s p}\left(\overline{t_{6} t_{2}}\right)=$ 3 , the algorithm places an RN, $s_{2}$, to the DFP of $T\left(t_{1}, t_{10}, t_{12}\right)$ and updates the $m s t$ by removing the edges $\overline{t_{1} t_{10}}$ and $\overline{t_{6} t_{2}}$, and adding $\overline{t_{1} s_{2}}, \overline{t_{10} s_{2}}$ and $\overline{t_{12} s_{2}}$ as illustrated in Figure 11(c). The rest of the execution steps of the algorithm is shown in Table2 and Figures 11(d) and (e). Finally the mst edges are steinerized to form SMT-MSPBEL in Figure 11(f).

\section{A Hybrid Approach}

In the previous sections we have presented two algorithms namely OTS-MST and IO-DT. Depending on the topology, both approaches may be superior to one another. For example in Figure 8 (c), the resulting topology of OTS-MST for a particular topology is illustrated where the algorithm required $20 \mathrm{RNs}$ to establish connectivity (i.e. the sum of sp-weights of the edges where the edge weights are annotated plus number of triangles in $M W I S$ ). In Figure11(f), however, for the same topology that is used in OTS-MST, the IO-DT required 19 RNs. In this example IO-DT outperforms OTS-MST in terms of the number of required RNs. The major advantage of IO-DT over OTS-MST is that unlike OTS-MST, IO-DT does not restrict itself to only the triangles having two mst edges and considers some triangles which do not have two mst edges as well. However, as illustrated in Figure 12, there are cases that some triangles might be in $\Gamma_{m s t}$ but not in $\Gamma_{d t}$ (i.e. $T(u, w, z) \in \Gamma_{m s t}$ and $\left.T(u, w, z) \notin \Gamma_{d t}\right)$.

In other words the triangle $T(u, w, z)$ is never considered in IO-DT. If

$$
\max \{G o T(u, w, z))+\operatorname{GoT}(v, u, w)\}>\max \{G o T(v, u, w)+G o T(v, w, z)\}
$$

where $\operatorname{GoT}(u, w, z)$ is the gain of triangle whose corners are $(u, w, z)$, then OTSMST would perform better than IO-DT.

The idea behind the hybrid solution is to combine the strengths of OTSMST and IO-DT, by executing these two algorithms sequentially. The order of 555 execution is important since the RNs, which are deployed by the first algorithm, 
Table 1: Execution of IO-DT algorithm

\begin{tabular}{|c|c|c|c|}
\hline Triangle $T_{i}$ & $W_{d f p}\left(T_{i}\right)$ & $W_{m s t}\left(T_{i}\right)$ & Action \\
\hline$\left(t_{7}, t_{9}, t_{10}\right)$ & 2 & 2 & skip \\
\hline$\left(t_{1}, t_{7}, t_{10}\right)$ & 2 & 2 & skip \\
\hline$\left(t_{2}, t_{8}, t_{12}\right)$ & 2 & 3 & $\begin{array}{l}\text { - Place } s_{1} \text { to DFP, Remove } \overline{t_{2} t_{8}} \text { and } \overline{t_{8} t_{12}} \text { from } m s t \\
\text { - Add } \overline{t_{2} s_{1}}, \overline{t_{8} s_{1}} \text { and } \overline{t_{12} s_{1}} \text { to } m s t\end{array}$ \\
\hline$\left(t_{1}, t_{10}, t_{12}\right)$ & 2 & 3 & $\begin{array}{l}\text { - Place } s_{2} \text { to DFP, Remove } \overline{t_{1} t_{10}} \text { and } \overline{t_{2} t_{6}} \text { from } m s t \\
\text { - Add } \overline{t_{1} s_{2}}, \overline{t_{10} s_{2}} \text { and } \overline{t_{12} s_{2}} \text { to } m s t\end{array}$ \\
\hline$\left(t_{6}, t_{11}, t_{15}\right)$ & 3 & 4 & $\begin{array}{l}\text { - Place } s_{3} \text { to DFP, Remove } \overline{t_{6} t_{15}} \text { and } \overline{t_{11} t_{15}} \text { from } m s t \\
\text { - Add } \overline{t_{6} s_{3}}, \overline{t_{11} s_{3}} \text { and } \overline{t_{15} s_{3}} \text { to } m s t\end{array}$ \\
\hline$\left(t_{3}, t_{8}, t_{15}\right)$ & 3 & 4 & $\begin{array}{l}\text { - Place } s_{4} \text { to DFP, Remove } \overline{t_{3} t_{8}} \text { and } \overline{t_{6} t_{14}} \text { from } m s t \\
\text { - Add } \overline{t_{3} s_{4}}, \overline{t_{8} s_{4}} \text { and } \overline{t_{15} s_{4}} \text { to } m s t\end{array}$ \\
\hline$\left(t_{2}, t_{6}, t_{15}\right)$ & 3 & 2 & skip \\
\hline$\left(t_{2}, t_{9}, t_{14}\right)$ & 3 & 3 & skip \\
\hline$\left(t_{4}, t_{5}, t_{13}\right)$ & 3 & 4 & $\begin{array}{l}\text { - Place } s_{5} \text { to DFP, Remove } \overline{t_{4} t_{13}} \text { and } \overline{t_{5} t_{13}} \text { from } m s t \\
\text { - Add } \overline{t_{4} s_{5}}, \overline{t_{5} s_{5}} \text { and } \overline{t_{13} s_{5}} \text { to } m s t\end{array}$ \\
\hline$\left(t_{2}, t_{9}, t_{10}\right)$ & 3 & 2 & skip \\
\hline$\left(t_{2}, t_{10}, t_{12}\right)$ & 3 & 2 & skip \\
\hline$\left(t_{3}, t_{8}, t_{12}\right)$ & 4 & 1 & skip \\
\hline$\left(t_{3}, t_{11}, t_{15}\right)$ & 4 & 2 & skip \\
\hline
\end{tabular}

may negatively affect the performance of the second algorithm and consequently the overall performance. Let $V$ be the set of terminals. The hybrid solution 


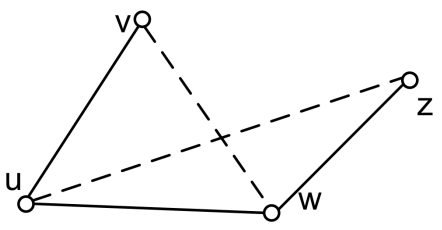

(a)

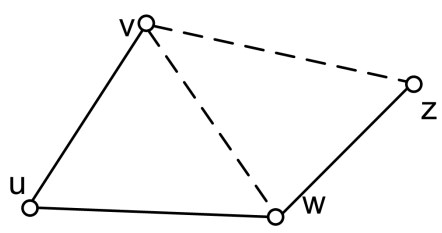

(b)

Figure 12: Illustrating the cases which is/is not handled by mst based triangulation and Delaunay Triangulation. Solid lines represent mst edges, dashed lines represent the triangulation. (a) The triangle $T(u, w, z)$ is considered but $T(v, w, z)$ is not considered in mst based triangulation. (b) $T(u, w, z)$ is not considered but $T(v, w, z)$ is considered in Delaunay triangulation.

Algorithm 4 HYBRID

Require: Set of terminal points $V$.

Ensure: Set of relay nodes $S$ and modified $G_{m s t}$
1: $\left\{S_{O T S-M S T}, G_{m s t}\left(V, E_{m s t}\right)\right\} \leftarrow \operatorname{OTS}-\operatorname{MST}(V)$
2: $\left\{S_{I O-D T}, G_{m s t}\left(V, E_{m s t}\right)\right\} \leftarrow \mathrm{IO}-\mathrm{DT}\left(V \cup S_{O T S-M S T}\right)$
3: $C_{1}=\left|S_{O T S-M S T}\right|+\left|S_{I O-D T}\right|+\sum_{e \in E_{m s t}} W_{s p}(e)$
4: $G_{\text {temp }}\left(V_{t e m p}, E_{\text {temp }}\right) \leftarrow G_{m s t}\left(V, E_{m s t}\right)$
5: $\left\{S_{I O-D T}, G_{m s t}\left(V, E_{m s t}\right)\right\} \leftarrow \operatorname{IO}-\mathrm{DT}(V)$
6: $\left\{S_{O T S-M S T}, G_{m s t}\left(V, E_{m s t}\right)\right\} \leftarrow$ OTS-MST $\left(V \cup S_{I O-D T}\right)$
7: $C_{2}=\left|S_{O T S_{M} S T}\right|+\left|S_{I O-D T}\right|+\sum_{e \in E_{m s t}} W_{s p}(e)$
8: if $C_{1}<C_{2}$ then
9: $\quad G_{m s t}\left(V, E_{m s t}\right) \leftarrow G_{t e m p}\left(V_{t e m p}, E_{\text {temp }}\right)$
10: end if
11: return steinerized $G_{m s t}$

compares the following two cases and selects the best order.

1. Run OTS-MST first and then run IO-DT: In this case the hybrid solution first runs the OTS-MST algorithm without steinerizing the edges in $E_{m s t}$ and then runs IO-DT. Let $S_{O T S-M S T}$ be the set of RNs deployed by OTS-MST. The algorithm determines $\Gamma_{d t}$ for $V \cup S_{O T S-M S T}$.

2. Run IO-DT first and then run OTS-MST: In this case the hybrid solution first runs the IO-DT algorithm without steinerizing the edges in $E_{m s t}$ and then runs OTS-MST. Let $S_{I O-D T}$ be the set of RNs deployed by IO-DT.

The algorithm determines $\Gamma_{m s t}$ for $V \cup S_{I O-D T}$.

The pseudo code of the hybrid approach is provided in Algorithm 4 in the appendix. We illustrate how the hybrid approach works with the same example 
that is used in figures 8 and 11] In this example we consider case (1) and first run OTS-MST algorithm. We leave out case (2), i.e., running IO-DT first, for space limitation and since it yields worse solution than case (1). The OTSMST algorithm deploys 4 RNs $S_{O T S-M S T}=\left\{s_{1}, s_{2}, s_{3}, s_{4}\right\}$ to the DFP's of the triangles in $M W I S$, as depicted in Figure 13(a). Then we apply IO-DT algorithm over the set of $V \cup S_{O T S-M S T}$ where $V$ is the set of terminals. The IO-DT algorithm deploys two more RNs $S_{I O-D T}=\left\{s_{5}, s_{6}\right\}$, as illustrated in Figure 13(b). Finally the algorithm steinerizes the mst edges in the resulting topology (Figure13(c)). As shown in this example the hybrid approach leverages the strengths of both OTS-MST and IO-DT and requires $18 \mathrm{RNs}$ to establish connectivity which outperforms both of them.

Theorem 3. The hybrid algorithm always performs better than (or equal to in the worst case) OTS-MST and IO-DT.

Proof. Both OTS-MST and IO-DT aims to improve the mst-based solution by leveraging the DFP based steinerization. In the worst case where no triangles is found to be optimized by DFP based steinerization, both algorithms end up with the steinerized mst. In other words running either OTS-MST or IO-DT never produces a topology which requires more RNs than mst-based solution. As described above, the hybrid approach compares two cases and selects the one which requires the fewest number of RNs. In the first case where OTS-MST is executed first, if IO-DT cannot find any more triangles to optimize then the result matches those of OTS-MST. Similarly in the second case where IO-DT is executed first, if OTS-MST cannot find any triangles, then the result will be equal to IO-DT. Since hybrid approach selects the best of the two cases, it will always perform better than (or equal to) IO-DT and OTS-MST.

\section{Analysis of the Algorithms} and IO-DT algorithms. We start with the following lemmas. 


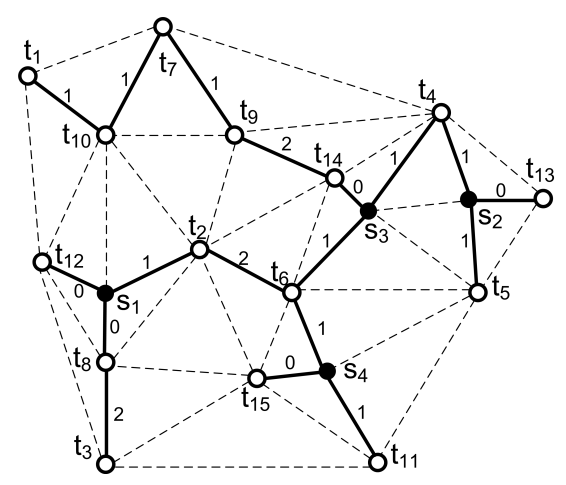

(a)

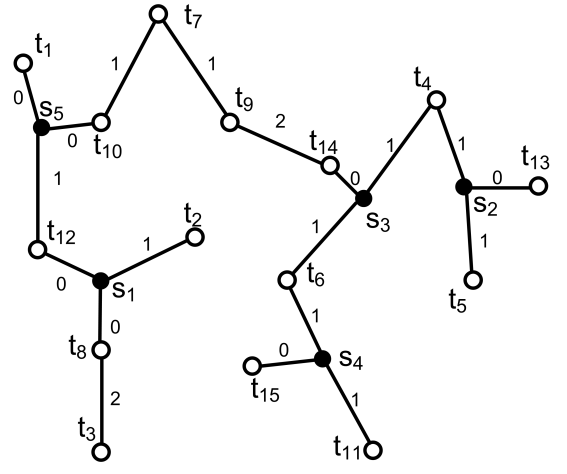

(b)

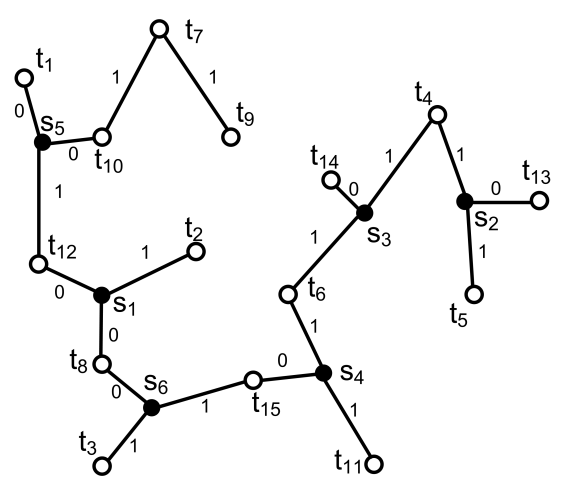

(c)

Figure 13: Illustrating how hybrid approach works. (a) The resulting $m s t$ after OTS-MST is applied. 4 RNs are placed. (b) First iteration of IO-DT is applied, the RN $s_{5}$ is placed. (c) Second iteration of IO-DT is applied, the RN $s_{6}$ is placed and $m s t$ is updated. Finally the mst edges will be steinerized as the last step as illustrated in Figure 11(f) 
Lemma 2. The mst is a sub-graph of DT [26].

Lemma 3. Both the number of edges and the number of triangles in DT can be bounded by $O(n)$ where $n$ is the number of terminals [27].

Corollary 1. Per lemma Q 2 , the mst can be computed after computing the Delaunay Triangulation of a set of terminals. The time complexity of finding Delaunay Triangulation is $O(n \log n)$ [27]. Using Kruskal's algorithm the cost of forming mst is $O(|E| \log |V|)[28]$. Per lemma 3, the number of edges for DT is $O(n)$ thus we can conclude that $|E|=O(n)$ and $|V|=n$. Therefore the cost of forming mst becomes $O(n \log n)$.

Lemma 4. The node degree (i.e., number of neighboring nodes) of a vertex in an Euclidean mst cannot be greater than 6 [29].

Lemma 5. Let $G_{T}\left(V_{T}, E_{T}\right)$ be the $T A G$ of a set terminals $V$ where the mst of $V$ is denoted as $G_{m s t}\left(V, E_{m s t}\right)$ and $|V|=n$. The number of vertices in $G_{T}$ (i.e., the number of triangles in mst triangulation) and the number of edges in $G_{T}$ (i.e., total number of triangular adjacencies) can be upper bounded by $O(n)$. In other words $\left|V_{T}\right|=O(n)$ and $\left|E_{T}\right|=O(n)$.

Proof. By definition 6, the triangles in mst triangulation contains two mst edges sharing one vertex where the shared vertex is non-leaf terminal in $G_{m s t}$. Let $v$ be a non-leaf terminal in $G_{m s t}$. Definition 6 implies that the number of triangles which can be formed by a pair of $m s t$ edges which share $v$, is a linear function of the node degree of $v$ (i.e. number of neighboring nodes). Therefore the total number of triangles in mst triangulation is a linear function of the sum of node degrees of non-leaf terminals. Per lemma 4 we know that the node degree of any node in $G_{m s t}$ can be at most 6 . Therefore, $\left|V_{T}\right|<6 n$, in other words $\left|V_{T}\right|=O(n)$. Similarly, the number of triangular adjacencies in mst triangulation is also a linear function of the sum of node degrees. Hence we have $\left|E_{T}\right|=O(n)$. 
Lemma 6. The cost of finding a path between two vertices in a tree is $O(|V|+$ $|E|)$, where $|V|$ and $|E|$ are the number vertices and edges, respectively. Since in an mst $|E|=|V|-1$, the time complexity of finding a path is $O(|V|)$ [28].

Theorem 4. The runtime complexity of OTS-MST algorithm is $O\left(n^{2}\right)$, where $n$ is the number of terminals.

Proof. The OTS-MST heuristic consists of a series of procedures. We will analyze each procedure individually. The algorithm computes the mst of terminals where the complexity is $O(n \log n)$ based on corollary 1 Next, $G_{T}\left(V_{T}, E_{T}\right)$ is formed. By lemma 5, we have $\left|V_{T}\right|=O(n)$ and $\left|E_{T}\right|=O(n)$. To form TAG, as described in section 4.1.1, the algorithm needs to consider every pair of edges in $m s t$. Since we have $\left|E_{T}\right|=O(n)$ the TAG can be computed in $O\left(n^{2}\right)$. After forming the TAG, the algorithm finds the set of edges which breaks cycles in TAG. Identifying these edges is by running Depth First Search (lines 5 - 7 in Algo. 2), where the cost is $O\left(\left|V_{T}\right|+\left|E_{T}\right|\right)=O(n)$. Then the algorithm computes MWIS (lines 8-9 in Algo. 22). In [25], it was proven that the cost of finding $M W I S$ is $O(n \log n)$. Since $M W I S \subset V_{T}$, then $|M W I S|=O(n)$. Hence the cost of finding adjacent triangles in MWIS is $O(n)$ and deploying relay nodes to the DFP of the triangles in MWIS is thus $O(n)$. Therefore the overall complexity is $O\left(n^{2}\right)$.

Theorem 5. The runtime complexity of IO-DT algorithm is $O\left(n^{2}\right)$, where $n$ is the number of terminals.

Proof. The IO-DT heuristic consists of a series of steps. We analyze each step individually. Throughout the proof we refer to the pseudo-code of Algorithm 3 in the appendix. IO-DT first calculates DT and mst of terminals where the cost of finding DT is $O(n \log n)$ [27] and the cost of forming mst using Kruskal's Algorithm is $O(n \log n)$ (by corollary 10). By lemma 3, since the number of 650 triangles in DT is $O(n)$, the cost of sorting the triangles becomes $O(n \log n)$ 
(line 4 in Algo. 3). The "for" loop (lines 5 to 24) iterates $O(n)$ times. Inside the loop, the algorithm calculates two paths $\Upsilon_{u v}$ and $\Upsilon_{u w}$ (line 6), and finds the two edges $e_{x}$ and $e_{y}$ having the largest sp-weights. By lemma 6, the cost of finding a path in a tree is $O(n)$. Thus the complexity of the "for" loop is $O\left(n^{2}\right)$. Hence the overall time complexity is $O\left(n^{2}\right)$.

Theorem 6. The runtime complexity of hybrid algorithm is $O\left(n^{2}\right)$, where $n$ is the number of terminals.

PROOF. As explained above the hybrid approach compares two possible cases and selects the best one. These cases are 1) Run OTS-MST first and then IO-DT, 2) Run IO-DT first and then OTS-MST. In either case the hybrid approach is indeed a sequential execution of IO-DT and OTS-MST approaches. By Theorems 4 and 5 we know that time complexities of OTS-MST and IODT are both $O\left(n^{2}\right)$, therefore the time complexity of the hybrid approach is $2\left(O\left(n^{2}\right)+O\left(n^{2}\right)\right)$ which is $O\left(n^{2}\right)$.

In the next section we validate the performance of our heuristics via simulation and show that their average performance surpasses all the best known deterministic algorithms for solving the SMT-MSPBEL problem.

\section{Performance Evaluation}

This section describes the experiment setup, performance metrics and simulation results.

\subsection{Experiment Setup and Baseline for Comparison}

A simulation environment is developed using Java. In the simulation, a number of terminals are randomly placed in a $1500 \mathrm{~m} \times 1500 \mathrm{~m}$ area. We compare the performance OTS-MST, IO-DT and the hybrid approach with two baseline algorithms, namely SMST [5, 10], and SMT-MSP 3-star [6]. In our simulations we assumed that the transmission ranges of terminals and RNs are the same. At this point we would like to note that in some practical applications of WSNs, 
the transmission ranges of terminals and relays can be different. If the transmission range of a terminal is greater than the transmission range of a $\mathrm{RN}$ then we do not need to change anything in our algorithms since the terminals can communicate with relays without any modification. However if the transmission range of a terminal is less than the transmission range of a relay we can still use the proposed algorithms with a possible small modification by placing an additional RN at the terminal locations in order to enlarge the transmission range of the terminal. In this case both our algorithms and the baseline algorithms will employ $N$ more relay nodes where $N$ is the number of terminals. For an arbitrary topology with $N$ terminals, let \#RN $N_{O T S-M S T}, \# R N_{I O-D T}, \# R N_{\text {hybrid }}$, and $\# R N_{3-s t a r}$ be the number of relay nodes required by OTS-MST, IO-DT, Hybrid, SMT-MSP and 3-Star algorithms respectively. If we have the following ordering,

$$
\# R N_{\text {hybrid }}<\# R N_{I O-D T}<\# R N_{O T S-M S T}<\# R N_{3-\text { star }}
$$

then the following is also true,

$\# R N_{\text {hybrid }}+N<\# R N_{I O-D T}+N<\# R N_{O T S-M S T}+N<\# R N_{3-\text { star }}+N$

where $N$ is the number of terminals. Therefore, for simplicity, assuming same transmission ranges for terminals and RNs does not have an effect on the performance of the algorithms.

675

In our simulations we varied two parameters, namely, number of terminals and transmission range of a $\mathrm{RN}(R)$. The $R$ is fixed at $100 \mathrm{~m}$ (both for terminals and RNs) to capture the effect of varying the number of terminals in the range $[10,50]$. When studying the impact of $R$, it is varied between $25 \mathrm{~m}$ to $200 \mathrm{~m}$ while fixing the number of terminals at 20 .

The following performance metrics are considered:

- Number of RNs: Minimizing the number of RNs for establishing the connectivity is the primary objective of the three proposed heuristics 
- Average Node Degree: This is the average number of neighbors of each $\mathrm{RN}$ in the resulting topology. A higher node degree indicates stronger connectivity and helps in balancing the traffic load and reducing the data latency.

\subsection{Performance Results}

This section provides the performance results. The result for each configuration is the average of 100 different random topologies. We observed that with a $\% 95$ confidence interval, our results stayed within $\% 6-\% 10$ of the sample mean.

Figure 14(a) shows that all of the three proposed heuristics perform significantly better than SMST and SMT-MSP 3-star in terms of the number of used RNs. The performance gap widens as the number of terminals increases reaching about $\% 20$ for a network of 50 terminals. This is mainly because the number of triangles considered by OTS-MST, IO-DT and the hybrid heuristic increases with the growth in terminal count. On the other hand, as expected the hybrid heuristic performs better than OTS-MST and IO-DT and the performance of IO-DT is slightly better than OTS-MST.

The performance comparison of our three heuristics is further illustrated in Figure 14(b), which shows the number of RNs gained by each algorithm relative to SMST. Clearly the gain for the hybrid approach is the highest among all three, which indicates that it requires fewer RNs than OTS-MST and IO-DT. The reason of such performance is that hybrid approach takes advantage of combining the strengths of both IO-DT and OTS-MST and maximizes the total gain. In addition, the results in Figure14(b) also show that the gain for IO-DT is higher than the gain for OTS-MST. The reason of the performance advantage of IO-DT over OTS-MST is basically the number of triangles considered by each algorithm. As mentioned earlier in the paper, IO-DT may consider triangles that do not have two mst edges, and thus factors in more triangles in the DFP optimization than OTS-MST which enables connecting the terminals with fewer RNs than OTS-MST. We repeated the experiments in Figure 14(a) and (b) for different values of $R$. We observed that for different transmission ranges the 


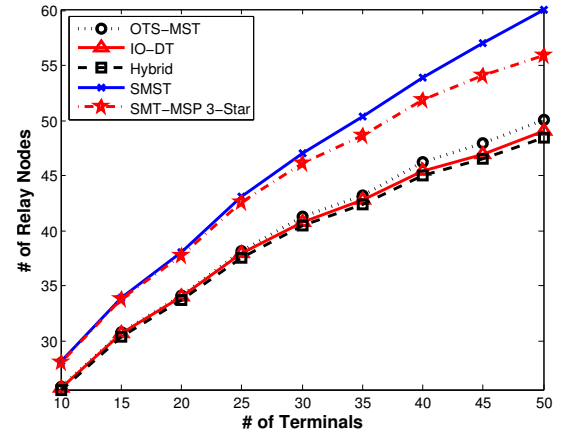

(a)

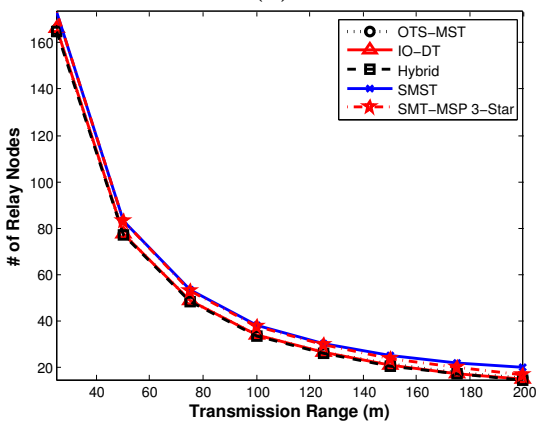

(c)

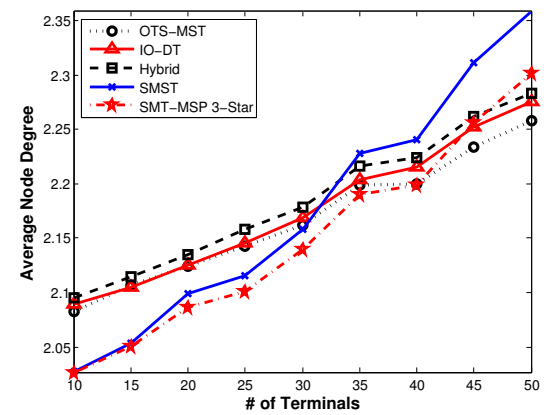

(e)

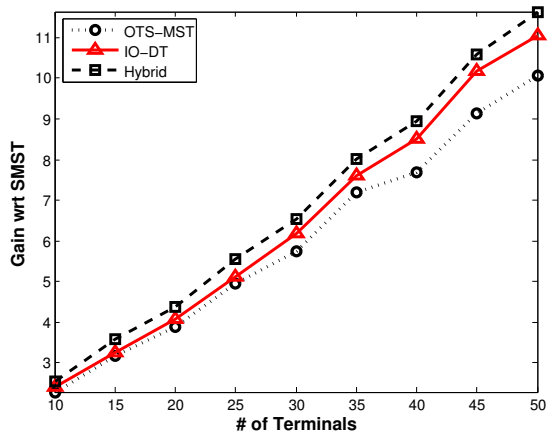

(b)

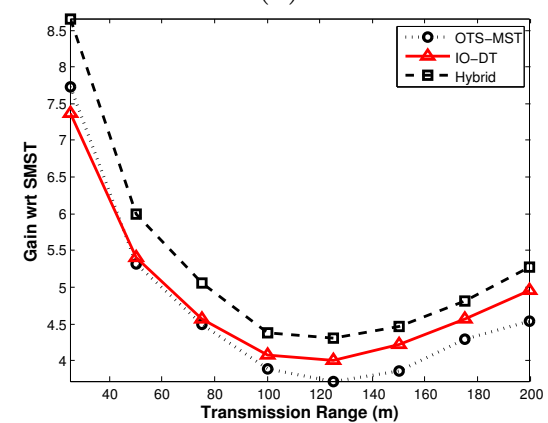

(d)

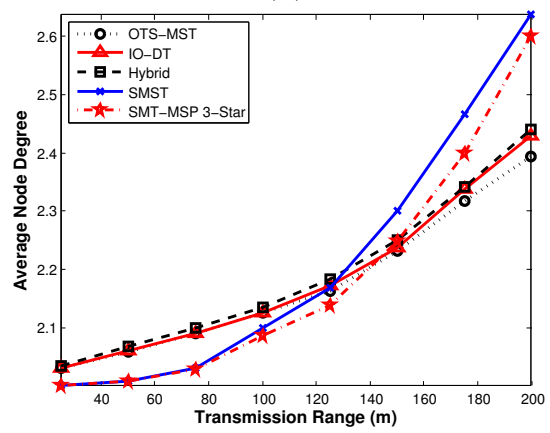

(f)

Figure 14: Under varying \# of terminals, comparison of (a) RN count, (b) gain with respect to SMST, (c) average node degree. Under varying transmission ranges, comparison of (d) RN count, (e) gain wrt. SMST, (f) average node degree. Note that for (a) and (d) a lower curve is better where for $(b)(c)(e)$ and (f) a higher curve is better.

effect of number of terminals on the required number of relays does not change and the pattern of each plot is the same.

Figure 14(c) illustrates the performance comparison in terms of number required RNs under varying $R$. The figure indicates that all three heuristics 
outperform SMST and SMT-MSP 3-star. It is clear that, the number of RNs diminishes as $R$ increases, since fewer RNs are required for steinerizing an edge for big $R$ values. In addition, the performance of the three heuristics is very close to one another under varying $R$. A better illustration of the performance comparison is depicted in Figure 14(d). Among the three heuristics, the hybrid approach always provided the highest gain and outperformed the others which is very much expected. We have further studied the effect of communication ranges on number of relay nodes for different number of terminals. We repeated the same experiment for $N=10, N=20$ and $N=30$ where $N$ is the number of terminals and observed that the pattern in each plot is the same which indicates the effect of transmission range to the required number of relay nodes is the same for different number of terminals.

The average node degree comparison of the algorithms is illustrated in Figures 14(e) and (f). In Figure 14(e), all three heuristics outperform the SMST and SMT-MSP 3-star algorithms when the number of terminals is small. In SMST and SMT-MSP 3-star algorithms almost all RNs have two neighbors since they are populated along mst edges. In contrast, all heuristics presented in this paper, place some of the RNs to the DFPs of the triangles, which yield a node degree of three. Thus if the algorithm finds more triangles to optimize, the average node degree gets closer to three, since more RNs will have a node degree of three. However, when the number of terminals exceeds 30, SMST and SMT-MSP 3-star start outperforming our proposed heuristics. This is mainly attributed to the fact that SMST and SMT-MSP 3-star requires more RNs than our heuristics for the same deployment area, and the increased RN density for large networks introduces more communication links.

Figure 14(f) compares the performance in terms of average node degree under varying transmission ranges. The average node degree of all algorithms increases as the transmission range grows. The reason is that for long transmission ranges, a relay can find more neighbors in its proximity which increases average node degree. For small transmission ranges, all three proposed heuristics perform better than SMST and SMT-MSP 3-star. Interestingly, however, 
the performance of SMST in terms of average node degree grows at a higher rate and outperforms the other algorithms when $R$ is bigger than $120 \mathrm{~m}$. The reason is that in OTS-MST, IO-DT and hybrid heuristics, RNs may be placed inside a triangle while in SMST RNs are always placed on the perimeter of a triangle. Due to this fact for big transmission ranges it is more likely for an RN in SMST to get connected with others on different edges and thus have more neighbors. Nonetheless, the increased RN count diminishes such an advantage for SMST and favors our heuristics.

\section{Conclusion}

In this paper, we have studied the problem of establishing connected topologies over wireless links through the placement of the least number of relays. This problem has significant importance in some WSN applications which operate unattended in harsh environments, e.g., battlefield surveillance and habitat monitoring. In this paper we have devised an optimal solution for the case of three terminals (i.e., WSN segments) by determining the Discrete Fermat Point (DFP), and used such a solution in developing three novel algorithms, namely OTS-MST, IO-DT, and the hybrid approach. OTS-MST applies DFP based steinerization to the triangles (i.e., sets of 3 terminals) having two minimum spanning tree (mst) edges and finds the subset of non-adjacent triangles which connects the terminals using the least number of relays. IO-DT computes the Delaunay Triangulation of terminals and iteratively checks if the DFP optimization of each triangle provides reduction in the total number of RNs as compared to an mst-based solution. After processing all triangles, the algorithm steinerizes the mst edges for both terminals and picked DFPs in order to form a connected topology. Finally we have presented a hybrid approach which combines the strengths of the OTS-MST and IO-DT. The simulation results have shown that all three heuristics significantly outperform competing schemes in the literature. In the future we plan to extend our work to establish connectivity in $3 \mathrm{D}$ settings. 


\section{Acknowledgment}

Work is supported by the NSF, award \# CNS 1018171.

\section{References}

780

785

[8] S. Lee, M. F. Younis, Optimized relay placement to federate segments in wireless sensor networks, IEEE Journal on Selected Areas in Communications 28 (5) (2010) 742-752.

[9] F. Senel, M. F. Younis, K. Akkaya, Bio-inspired relay node placement

[1] I. F. Akyildiz, W. Su, Y. Sankarasubramaniam, E. Cayirci, Wireless sensor networks: a survey, Computer Networks 38 (4) (2002) 393-422.

[2] M. F. Younis, S. Lee, I. F. Senturk, K. Akkaya, Topology Management Techniques for Tolerating Node Failure, Springer, 2012.

[3] M. Younis, I. F. Senturk, K. Akkaya, S. Lee, F. Senel, Topology management techniques for tolerating node failures in wireless sensor networks: A survey, Computer Networks 58 (0) (2014) 254 - 283. doi:http://dx.doi.org/10.1016/j.comnet.2013.08.021.

[4] M. F. Younis, K. Akkaya, Strategies and techniques for node placement in wireless sensor networks: A survey, Ad Hoc Networks 6 (4) (2008) 621-655.

[5] G.-H. Lin, G. Xue, Steiner tree problem with minimum number of steiner points and bounded edge-length, Inf. Process. Lett. 69 (2) (1999) 53-57.

[6] X. Cheng, D.-Z. Du, L. Wang, B. Xu, Relay sensor placement in wireless sensor networks, Wireless Networks 14 (3) (2008) 347-355.

[7] E. L. Lloyd, G. Xue, Relay node placement in wireless sensor networks, IEEE Trans. on Comp. 56 (2007) 134-138.

heuristics for repairing damaged wireless sensor networks, IEEE Trans. on Vehicular Techn. 60 (4) (2011) 1835-1848. 
[10] D. Chen, D.-Z. Du, X.-D. Hu, G.-H. Lin, L. Wang, G. Xue, Approximations for steiner trees with minimum number of steiner points, Theor. Comput. Sci. 262 (1) (2001) 83-99.

[16] S. Kimence, I. Bekmezci, Weighted relay node placement for wireless sensor network connectivity, Wireless Networks 20 (4) (2014) 553-562. doi:10.1007/s11276-013-0624-7.

[17] K. Xu, Q. Wang, H. S. Hassanein, G. Takahara, Optimal wireless sensor 825

[18] Q. Wang, G. Takahara, H. S. Hassanein, K. Xu, On relay node placement and locally optimal traffic allocation in heterogeneous wireless sensor networks, in: LCN, 2005, pp. 656-664. 
[19] Q. Wang, K. Xu, G. Takahara, H. S. Hassanein, Locally optimal relay node placement in heterogeneous wireless sensor networks, in: GLOBECOM, 2005 .

[20] Q. Wang, K. Xu, H. S. Hassanein, G. Takahara, Minimum cost guaranteed lifetime design for heterogeneous wireless sensor networks (wsns), in:

[21] K. Xu, H. S. Hassanein, G. Takahara, Q. Wang, Relay node deployment strategies in heterogeneous wireless sensor networks: multiple-hop communication case, in: Proc. of SECON, 2005.

[22] S. Li, G. Chen, W. Ding, Relay node placement in heterogeneous wireless

[25] S. Pawagi, Maximum weight independent set in trees, BIT 27 (2) (1987) 170-180.

[26] F. Aurenhammer, Voronoi diagrams - a survey of a fundamental geometric data structure, ACM Comput. Surv. 23 (3) (1991) 345-405.

[27] M. de Berg, M. van Kreveld, M. Overmars, O. Schwarzkopf, Computational Geometry: Algorithms and Applications, 2nd Edition, Springer-Verlag, 2000.

[28] T. H. Cormen, C. E. Leiserson, R. L. Rivest, C. Stein, Introduction to Algorithms, Second Edition, The MIT Press, 2001. 
${ }_{855}$ [29] X.-Y. Li, Y. Wang, W.-Z. Song, Applications of k-local mst for topology control and broadcasting in wireless ad hoc networks, IEEE Trans. Parallel Distrib. Syst. 15 (12) (2004) 1057-1069. 


\section{APPENDIX}

Table 2: Notations and Definitions

\begin{tabular}{|c|c|}
\hline Notation & Definition \\
\hline$W_{s p}(u, v)=\left\lceil\frac{|u v|}{R}\right\rceil-1$ & $\begin{array}{l}\text { sp-weight of }(u, v) \text { : minimum number of relay nodes } \\
\text { for connecting two terminals } u \text { and } v \text { (see Defini- } \\
\text { tion 1) }\end{array}$ \\
\hline$T_{i}(u, v, w)$ & Any arbitrary triangle whose vertices are $(u, v, w)$ \\
\hline$W_{f p}\left(T_{i}, q\right)$ & $\begin{array}{l}\text { The number of relay nodes required for connect- } \\
\text { ing the vertices of } T_{i} \text { to an arbitrary point } q \text {. } \\
W_{f p}\left(T_{i}, q\right)=\left\lceil\frac{|u q|}{R}\right\rceil+\left\lceil\frac{|v q|}{R}\right\rceil+\left\lceil\frac{|w q|}{R}\right\rceil-2 \text { (see Defi- } \\
\text { nition [3) }\end{array}$ \\
\hline$W_{f p}\left(T_{i}, \varphi_{i}\right)$ or $W_{d f p}\left(T_{i}\right)$ & $\begin{array}{l}\text { The Discrete Fermat Point weight (dfp-weight) of } T_{i} \text {, } \\
\text { where } \varphi_{i} \text { is the best point at which the vertices of } T_{i} \\
\text { can be connected. }\end{array}$ \\
\hline$W_{m s t}\left(T_{i}\right)$ & mst-weight of $T_{i}$, (see Definition 8 and Figure 10) \\
\hline $\operatorname{Gain}\left(T_{i}\right)$ & $\begin{array}{l}\text { The difference between mst-weight and df-weight } \\
W_{m s t}\left(T_{i}\right)-W_{d f p}\left(T_{i}\right)\end{array}$ \\
\hline$G_{m s t}$ & Minimum Spanning Tree of set of terminals $V$ \\
\hline$\Gamma_{m s t}$ & $\begin{array}{l}\text { mst-triangulation of a set of terminals } V \text { (see Defini- } \\
\text { tion } 6\end{array}$ \\
\hline$\Gamma_{d t}$ & Delaunay triangulation of a set of terminals $V$ \\
\hline
\end{tabular}

